\title{
Precision Health Data: Requirements, Challenges and Existing Techniques for Data Security and Privacy
}

\author{
Chandra Thapa and Seyit Camtepe \\ CSIRO Data61, Australia \\ \{chandra.thapa, seyit.camtepe\}@data61.csiro.au
}

\begin{abstract}
Precision health leverages information from various sources, including omics, lifestyle, environment, social media, medical records, and medical insurance claims to enable personalized care, prevent and predict illness, and precise treatments. It extensively uses sensing technologies (e.g., electronic health monitoring devices), computations (e.g., machine learning), and communication (e.g., interaction between the health data centers). As health data contain sensitive private information, including the identity of patient and carer and medical conditions of the patient, proper care is required at all times. Leakage of these private information affects the personal life, including bullying, high insurance premium, and loss of job due to the medical history. Thus, the security, privacy of and trust on the information are of utmost importance. Moreover, government legislation and ethics committees demand the security and privacy of healthcare data. Besides, the public, who is the data source, always expects the security, privacy, and trust of their data. Otherwise, they can avoid contributing their data to the precision health system. Consequently, as the public is the targeted beneficiary of the system, the effectiveness of precision health diminishes. Herein, in the light of precision health data security, privacy, ethical and regulatory requirements, finding the best methods and techniques for the utilization of the health data, and thus precision health is essential. In this regard, firstly, this paper explores the regulations, ethical guidelines around the world, and domain-specific needs. Then it presents the requirements and investigates the associated challenges. Secondly, this paper investigates secure and privacy-preserving machine learning methods suitable for the computation of precision health data along with their usage in relevant health projects. Finally, it illustrates the best available techniques for precision health data security and privacy with a conceptual system model that enables compliance, ethics clearance, consent management, medical innovations, and developments in the health domain.
\end{abstract}

Keywords: Precision health, legal requirements, ethical guidelines, security, privacy, artificial intelligence

\section{Introduction}

Precision health is a precise, personalized, prescriptive, and preventive approach to healthcare. As illustrated in Figure 1 it leverages collective information from diverse sources, including omics (e.g., genomics), lifestyle, environment, social media, internet of medical things, medical history, pharmaceuticals, and medical insurance claims [12. Precision health will not only refine the current health care practices of providing care after an illness, but also predict, prescribe, and prevent the illness before they develop. For example, the risk of type 2 diabetes mellitus is identified through longitudinal study (8 years) of the clinical measures and tests, including omics profiling, microbiome, and wearable monitoring [3. In another work, online review data of restaurants on social media are leveraged to predict the hygiene of the restaurant and health risks [4. People can take advantage of these predictions at the right time to avoid potential health risks. Besides, the preventive approach (e.g., detection and treatment of illness at early stages) and precision diagnosis (e.g., right drugs and correct diagnosis) in precision health enables a reduction in the healthcare cost, which is expensive and ever increasing 5 . For example, the USA spent $\$ 3.6$ trillion in 2017, which is 4.4\% higher than in 2017 [6]. Similarly, Australia spent $\$ 181$ billion on health care in 2016-17, which is $1.6 \%$ higher than the average over 2011-2015[7].

The main fuel of precision health for its operation is health data, which is growing at a fast pace. The growth is due to electronic health records (EHR), medical images, and the internet of medical things (IoMT), including wearable devices (e.g., fitness trackers such as FitBits). It is estimated that 2,314 exabytes of health data will be produced in 2020 [8. These data are profiling individuals, and it can be leveraged by clinicians and researchers in the precision health ecosystem. Usually, these data are decentralized in nature and non-iid in characteristics.

The precision health data has mainly seven stages in its life-cycle, namely data generation, collection, processing (e.g., health data cleaning and data encryption), storage, management (e.g., creating metadata and access control), analytics and inference. Data analytics is an integral part of precision health. It is a systematic use of data combined with quantitative as well as qualitative analysis to make decisions 9]. It supplies techniques to transform 


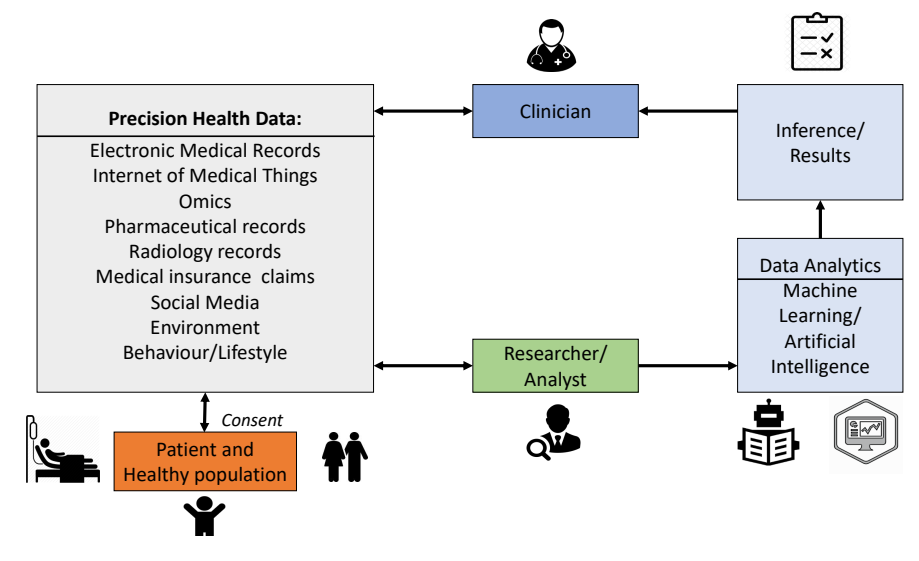

Fig. 1: Precision health ecosystem

accumulated raw data into valuable insights, and their utilization enables an evidence-based healthcare delivery. For example, mutation prediction [10. Artificial Intelligence (AI) and Machine learning (ML) boost analytic, and health data analytics have been a part of healthcare [11|12. The analytic has a huge impact on medical research, daily life, patient experience, ongoing care, prediction, and prevention [1314]. Besides, it is a growing industry [15]. It saves a considerable amount of expenditure in the healthcare economy. It is estimated that the key clinical health AI applications can save $\$ 150$ billion annual savings for the USA healthcare economy by 2026 [16].

Based on the precision health data life-cycle, we can broadly divide health data into three categories, namely data-at-rest (stored, not currently transmitted or processed), data-in-transit (data currently being transferred from one part to another) and data-in-use (data in memory, including CPU caches and registers). Data analytics predominantly deals with data-in-use. Although precision health has the potential to revolutionize current healthcare, it faces difficulties due to security, privacy, ethical and legal concerns related to health data of all categories. It contains sensitive information of patient and carer, including their identity, medical condition of the patient, and cure. A proper consent needs to be taken for the use and reuse of the data from their owner. In addition, care must be taken during all stages of the data life cycle because leakage of this information affects personal life, including poor social networking and loss of job due to the medical history, no employment, and high insurance premium. Patient engagement survey 2018 by MedicalDirector in partnership with HotDoc [17] found that $93 \%$ and 91\% Australian rate security and privacy, respectively, are a top concern. This concern is not limited to Australians; it is everywhere. Besides, among all data breaches, health data covers a significant portion, and it is increasing [18]19]20|21. Governments are also concerned about the security and privacy of health data. They have been regulating and managing the concerns through government policies and legislation (refer to Section 2.1 for details).

We refer the sensitive information to protected health information or personal information, which are defined in the following. These terms refer to the same information in the health domain, so we refer to them by simply personal information (PI) in the remainder of this paper for convenience.

Protected health information: According to the health insurance portability and accountability act (HIPPA) of USA, protected health information (PHI) includes all individually identifiable information, including demographic data, medical histories, test results, insurance information, other information used to identify a patient or provide healthcare services or healthcare coverage [22].

Personal information: Based on Privacy Act 1988, Australia, personal information (PI) is information or an opinion that identifies you or could identify you and includes information about your health [23. In the European Union's general data protection regulation (GDPR) [24, personal information is defined as any data that relates to an identified or identifiable individual. This data includes online identifiers (e.g., IP address), sales databases, location information, CCTV footage, bio-metric data, loyalty scheme records, and health information.

The current legal and ethical aspects are important for health data, which includes PI. For precision health data security, privacy and trust, there is no elaborative work that investigates these aspects to identify the requirements and challenges along with recently evolving enabler techniques, including privacy-preserving distributed collaborative machine learning techniques. The requirements guide to maintain compliance, and the techniques ensure it in precision health. Refer to Table 1 for related works. 
Table 1: Related works in health data security and privacy

\begin{tabular}{|c|c|c|c|}
\hline Reference & Focus & Review & Technology focus \\
\hline 25 & $\begin{array}{l}\text { Electronic health record } \\
\text { system }\end{array}$ & $\begin{array}{l}\text { Compliance, information systems acquisition } \\
\text { development, access control, communications } \\
\text { and operations management, human resource } \\
\text { security }\end{array}$ & $\begin{array}{l}\text { Pseudo-anonymity technique, encryption, } \\
\text { authentication systems, audit logs }\end{array}$ \\
\hline 26] & e-Health clouds & $\begin{array}{l}\text { Requirements for e-health cloud, } \\
\text { privacy-preserving approaches }\end{array}$ & $\begin{array}{l}\text { Various cryptography based approaches, } \\
\text { including homomorphic encryption and } \\
\text { searchable encryption }\end{array}$ \\
\hline 27$]$ & Big data in healthcare & $\begin{array}{l}\text { Data protection law of seven countries (e.g., } \\
\text { HIPAA of USA and Data Protection } \\
\text { Directives of EU) }\end{array}$ & $\begin{array}{l}\text { Authentication, encryption, } \\
\text { de-identification, access control }\end{array}$ \\
\hline 28 & Biomed data science & $\begin{array}{l}\text { Brief discussion on HIPAA, research ethics, } \\
\text { and patient's viewpoint }\end{array}$ & $\begin{array}{l}\text { De-identification methods, data } \\
\text { anonymization methods, privacy- } \\
\text { preserving predictive modeling } \\
\text { (use cases of federated learning and } \\
\text { encrypted data analysis) }\end{array}$ \\
\hline 29] & Big healthcare data & $\begin{array}{l}\text { Briefly mentioned salient features of data } \\
\text { protection laws of nine countries (e.g., HIPAA } \\
\text { USA, Data projection directive EU) }\end{array}$ & $\begin{array}{l}\text { Authentication, encryption, data masking, } \\
\text { access control, monitoring and auditing, } \\
\text { de-indentification, HybrEx }\end{array}$ \\
\hline 30 & eHealth cloud security & $\begin{array}{l}\text { US standards (e.g., HIPAA), International } \\
\text { Standards (e.g., ISO/IEC 27000-series), } \\
\text { GDPR EU }\end{array}$ & Patient-centric approach, encryption \\
\hline 31 & Federated learning & $\begin{array}{l}\text { Current progress on federated learning } \\
\text { (technical) attacks and defenses }\end{array}$ & $\begin{array}{l}\text { Federated learning, secure multi-party } \\
\text { computation, differential privacy }\end{array}$ \\
\hline 32 & Human genomic data & $\begin{array}{l}\text { Privacy/security problems related to genomic } \\
\text { data sharing/computation }\end{array}$ & $\begin{array}{l}\text { Homomorphic encryption, Intel SGX, } \\
\text { differential privacy }\end{array}$ \\
\hline 33 & Healthcare & $\begin{array}{l}\text { Challenges for the machine learning techniques, } \\
\text { including vulnerabilities in machine learning } \\
\text { pipeline, model training, adversarial machine } \\
\text { learning and privacy-preserving machine } \\
\text { learning (e.g., federated learning in brief), } \\
\text { countermeasures against adversarial attacks } \\
\text { on models, responsible machine learning }\end{array}$ & $\begin{array}{l}\text { Machine learning applications on } \\
\text { prognosis, diagnosis, treatment }\end{array}$ \\
\hline 34 & Medical imaging data & $\begin{array}{l}\text { Overview of methods for federated, secure and } \\
\text { privacy-preserving artificial intelligence }\end{array}$ & $\begin{array}{l}\text { Re-identification issue, federated machine } \\
\text { learning, differential privacy, homomorphic } \\
\text { encryption, secure multi-party computation, } \\
\text { secure hardware implementation (in brief) }\end{array}$ \\
\hline 35] & $\begin{array}{l}\text { Distributed learning in } \\
\text { health care }\end{array}$ & Legal context in brief & $\begin{array}{l}\text { Machine learning techniques, distributed } \\
\text { (deep) learning, block-chain }\end{array}$ \\
\hline This paper & Precision Health data & $\begin{array}{l}\text { Detailed requirements based on regulations } \\
\text { (e.g., GDPR EU), ethical guidelines, and } \\
\text { health domain, techniques for (health) data } \\
\text { security and privacy, and its consideration } \\
\text { in notable health projects }\end{array}$ & $\begin{array}{l}\text { Machine learning in healthcare, No-peek } \\
\text { learning, including federated and split } \\
\text { learning on medical informatics, data-centric } \\
\text { solutions, cryptography, access control, } \\
\text { anonymization, pseudonymization, hardware } \\
\text { based security and privacy, homomorphic } \\
\text { encryption, multiparty computation, } \\
\text { differential privacy }\end{array}$ \\
\hline
\end{tabular}

\subsection{Our contributions}

The precision health $(\mathrm{PH})$ data is usually isolated and distributed (e.g., data stored at different hospitals), and it comes from a diverse field. In light of barriers, as mentioned earlier, including security and privacy, it is important to explore the best ways for health data handling and use, including breaking the precision health data silos required to leverage $\mathrm{AI} / \mathrm{ML}$ efficiently. In this regard, this paper thoroughly explores the requirements, lists out the challenges, and presents the potential candidate methods that enable data privacy and security. Firstly, this paper, in Section 2, surveys the data regulations and ethical guidelines from data security and privacy perspectives. 
This provides detailed requirements for compliance whilst handling PH data. Then, considering the sensitivity of $\mathrm{PH}$ data in health decision making, this paper studies the requirements for data trustworthiness. Afterward, based on these requirements, it highlights the existing challenges related to $\mathrm{PH}$ data in Section 3. Secondly, in Section 4 . it presents current techniques for $\mathrm{PH}$ data security and privacy. As the computing environment may not be a trusted platform, PH data privacy and security whilst computation need to be addressed. So, this paper presents the machine learning paradigms and healthcare, including the state-of-the-art privacy-by-design machine learning approaches that ensure PH data privacy and security, in Section 5.2. Together with the relevant health projects, and their PH data security and privacy techniques in Section 6, the candidate techniques are illustrated with a conceptual system model for the precision health platform to provide an overall picture in Section 7.1 .

\section{Requirements for precision health data privacy, security and trust}

Precision healthcare is a data-driven healthcare approach. Thus compliance, both to law and ethics, while handling the health data is of utmost importance to avoid penalties and maintain trustworthiness. The proper requirements for privacy, security, and trust of the precision health $(\mathrm{PH})$ data enable us to design and maintain the compliancefriendly techniques and trustworthy platform to handle the $\mathrm{PH}$ data. In this regard, we extract the requirements due to law and ethics in the following sections.

Firstly, we revisit the general definition of and the distinction between law and ethics. According to the Oxford dictionary, the law is the system of rules which a particular country or community recognizes as regulating the actions of its members and which it may enforce by the imposition of penalties [36. Law has a set of rules and regulations with legal binding. A government governs it. On the other hand, ethics are moral principles that govern a person's behavior or the conducting of activity [37. Ethics has a set of guidelines (e.g., code of conduct) governed by individuals, legal and professional norms. It guides us on good and evil, or right and wrong in all aspects of human affairs. Violations of ethical standards result in penalties, including job termination, monetary fines, and legal actions. Ethics and law are complementary to each other, and both are required for better judgment and decision.

In order to provide a comprehensive requirement as much as possible, the literature is searched and filtered based on its contents and source. For laws (e.g., federal laws) and ethics, relevant documents from governments and institutions are selected that are inclusive and can provide an overall overview. Search is done in the various search engines, including Google Scholar, Scopus, and PubMed, by using keywords, including privacy, security, trust in health data, ethics, and ethical requirements. Many sources have overlapping issues and requirements. Thus, we consider only those which cover most of them. Also, review papers discussing the major laws and ethical requirements for the related field are considered.

\subsection{Requirements due to law}

There have been significant initiations from several countries and organizations towards the acts/regulations of health data privacy, security, and trust. These are introduced to ensure the privacy of personal information (PI), which is more relevant when it comes to $\mathrm{PH}$ data. To understand the current legislation that provides baseline security and privacy rules around the world, we state the regulations in some countries where EHR is commonly used as an illustration. This includes the USA, EU, and Australia. Broad coverage of regulations around the world is not the scope of this paper. Even within one country, their states can have their separate privacy legislation. For example, states have different general privacy legislation in Australia [38, and similarly in the USA, e.g., California Consumer Privacy Act [39. However, the requirements are standard and similar.

HIPAA: The USA has a health insurance portability and accountability act (HIPAA) enacted in 1996 for the privacy and security of healthcare information, including health data. The privacy rule standards of HIPAA address the use and disclosure of health data with PI. It assures the protection of PI while allowing the flow of health information (e.g., electronic exchange), which is highly required for medical decisions and well-being [40. HIPAA privacy rules apply to health plan $\mathbb{1}^{1}$, health care clearinghouses ${ }^{2}$, and to health care providers (e.g., hospitals, physicians, dentists, and other practitioners) who transmits health information. HIPAA does not apply to deidentified data, which refers to the data set of the individual from which the PI cannot be traced even by linking

\footnotetext{
${ }^{1}$ Health plans are individuals or group plans that provide or pay the cost of medical care. For example, health insurers, Medicare, health maintenance organizations, and long-term care insurers [40].

${ }^{2}$ Health care clearinghouses are entities that process nonstandard information they receive from another entity into a standard or vice versa. For example, billing services, repricing companies, and community health management information systems [40].
} 
with other available data sets. For secondary use of data including research, and analytics, it is mandatory to obtain written authorization from the patients.

The security standards of HIPAA address the protection of health information that is held or transferred in electronic form. The main aim of the standards is to protect the privacy of the PI while allowing authorized entities to access and process data. The security standard applies to health plans, health care clearinghouses, and to any health care provider who transmits health information. The security rule states that the PI must be confidentia ${ }^{3}$, integra 4 , and availabl 5 . The PI holders must identify and protect against threats to the security and integrity of the information and protect from any impermissible uses or disclosures [41. The security standard of HIPAA provides guidelines for the following safeguards along with non-compliance penalties:

1. Administrative safeguards, including (i) security management process having risk analysis, risk management, sanction policy, and information system activity review, (ii) information access management having isolating health care clearinghouse functions, and access authorization, (iii) contingency plan having data backup plan, disaster recovery plan, and emergency mode operation plan.

2. Physical safeguards, including device and media controls with the implementation of disposal and media re-use provisions.

3. Technical safeguards, including (i) access control having unique user identification, emergency access procedure, and encryption and decryption, (ii) audit controls having record and examine activity, and (iii) integrity control having mechanism to authenticate electronically protected health information.

The HIPAA breach notification rule requires to provide notification following a breach of protected health information to the affected individuals. To strengthen the data protection requirements, there are other regulations along with HIPAA in the USA. These regulations include (a) Genetic Information Non-discrimination Act (GINA, [42]), and (b) Health Information Technology for Economic and Clinical Health (HITECH, [43]). GINA was enacted in 2008, and it addresses the issues related to the discrimination based on genetic information, whereas HITECH was enacted in 2009, and it addresses the issues associated with electronic health records and health technologies. These regulations strengthen consumers' information rights on their data and prohibit disclosure of health information without their consent except for treatment, payment, or health care operations.

GDPR: General Data Protection Regulation (GDPR) 24] is the latest EU's data protection law. It has been in effect in EU since May 2018 to protect PI and harmonize data privacy laws across Europe and European Economic Area (EEA). It also regulates the PI data sharing outside EU and EEA. The GDPR applies to any organization that collects or processes PI of EU residents. It has the following six key principles [24]:

1. Lawfulness, transparency and fairness while handling the PI. The organizations are obliged to inform the individual about the process of data handling transparently.

2. The purpose of PI shall be specified, explicit, and legitimate. Re-using the data for other purposes than the original one is restricted.

3. The data storage and collection of PI shall be minimized to that which is enough and relevant.

4. The stored or collected data shall be accurate and up to date (by erasing or rectifying if the data is inaccurate).

5. The period of storing the PI shall be limited to its necessity of the original purpose. It should be deleted once it is not necessary.

6. PI shall be processed in a secure manner, including protection against unauthorized or unlawful processing and accidental loss or damage. It is required that the data protection is "by design" and "by default". Privacyby-design in data protection requires all safeguards necessary to ensure compliance with the regulation key principles since the first phases of relevant design and creation. On the other side, data protection by-default requires all steps to prevent unnecessary collection and processing of personal data other than needed for the purpose.

GDPR empowers EU citizens by providing the right to access their PI, withdraw their consent at any time, ask to erase data, right to restrict processing, and right to be notified if their data is breached within 72 hours. Moreover, it also addresses the issues that can come due to the rise of ML algorithms in data processing. GDPR requires the explanations of the algorithmic outcomes before its implementation. Under GDPR, if the organizations do not comply with its regulations, then there is a provision of maximum penalties, including a fine that will be greater of $€ 20$ million or four percent of an organization's annual global revenue. EU has been working on Ethics for Artificial

${ }^{3}$ Confidentiality of PI means that the information is not available or disclosed to unauthorized person [41].

${ }^{4}$ Integrity of PI means that the information is not altered or destroyed in an unauthorized manner 41].

${ }^{5}$ Availability of PI means that the information is accessible and usable on demand by an authorized person 41. 
Intelligence, which includes fairness principle, transparent, intelligible, and responsible AI system, guaranteeing privacy by default, and by design [44].

Australia Privacy Act: In Australia, the Privacy Act 1988 [45] guides the privacy and security framework for PI. The Privacy Act applies to most Commonwealth government agencies (including tax office and department of human services), all private sector organizations that have an annual turnover of more than three million dollars, and some other organizations that meet particular criteria, for example, health service providers. Under the privacy act, the Australian Information Commissioner makes guidelines, known as the Australian Privacy Principles (APPs) [46]. There are thirteen basic APPs, which are grouped into the following five parts.

1. For the consideration of PI privacy, there are two APPs. APP 1 outlines the requirements to manage PI openly and transparently. The organizations must have a clearly expressed and up to date privacy policy and complaints procedure. In addition, they must ensure their compliance with APPs. APP 2 states that the individuals must have an option of dealing anonymously or use pseudonym 6 where possible.

2. For the collection of PI, there are three APPs. APP 3 outlines that an organization can collect PI when it is reasonably necessary for, or directly related to, the organization's function or activities. The collection must be done lawfully and by fair means (with consent). APP 4 outlines the steps to take if the organizations receive unsolicited PI (collected without asking individuals). If the unsolicited PI can be collected under APP 3 and not in a Commonwealth record, then it must be destroyed or de-identified as soon as practicable. APP 5 outlines the information that must be provided to an individual when their data is being collected. This includes the organization's APP policy, detail about the organization such as contact details, purpose of the collection, complaint handling process, and potential overseas disclosure.

3. To deal PI, there are four APPs. APP 6 deals with the use and disclosure of PI. The PI can only be used or disclosed for the purpose it was collected or for a secondary use if an exception applies including consent for the secondary use, provide health services, and authorize by Australian law. APP 7 prohibits organizations from using or disclosing PI for direct marketing unless an exception applies. Direct marketing involves the use of PI to promote goods and services. If the organizations are allowed to use PI for direct marketing, then they must always allow individuals for an "opting out" option (not to receive direct marketing). Further, they must provide the source of PI to individuals upon request unless it is impracticable to do so. APP 8 introduces an accountability approach for cross-border disclosure. The organizations must ensure the overseas recipients follow APPs; otherwise, they may hold accountable for their recipient's breach. APP 9 restricts the adoption, use, and disclosure of government related identifier: 7 by organizations.

4. For the integrity of PI, there are two APPs. APP 10 requires organizations to ensure PI they collect, use, or disclose are accurate, up-to-date, complete, and relevant. APP 11 states that the organizations must take reasonable steps to protect PI they hold from misuse, interference, loss, unauthorized access, and modification, or disclosure (including hacking).

5. To access and correct PI, there are two APPs. APP 12 requires PI holding organizations to provide individual access on request. It also sets out procedures for acceptance or rejection of the request. APP 13 states that the organizations must correct their data if it is wrong, or if an individual requests correction. This is required to ensure data accuracy, completeness, and relevancy.

Besides the Privacy Act, there is my health records act 2012 [47] in Australia. This act provides a legal framework for the management of my health record system, which provides an individual's key health information to the healthcare recipient. The My Health Records act follows the APPs for the collection, use, and disclosure of health information, including my health records. Besides, the Notifiable Data Breaches (NBD) scheme is commenced from February 2018 in Australia. Under this scheme, regulated entities (e.g., Australian Government agencies, business organizations with an annual turnover of $\$ 3$ million or more, health service providers and other organizations) require to notify affected individuals and the Australian Information Commissioner about the breaches that can harm one or more individuals. The overall summary of the requirements due to regulations is stated in Table 2

For all countries besides their laws, there are binding international laws such as the universal declaration of human rights and the European convention on human rights [48]. These laws stress on the privacy of the individuals (e.g., PI), and their requirements are covered above.

${ }_{7}^{6}$ A pseudonym is a name, term or descriptor that is different to an individual's actual name [46].

${ }^{7}$ An identifier is a number, letter or symbol, or combination of any or all of those things, that is used to identify the individual [46]. 
Table 2: Summary of requirements due to regulations

\begin{tabular}{|c|c|c|}
\hline Country & Requirements & Overall requirements \\
\hline $\begin{array}{c}\text { USA } \\
\text { (HIPAA [4041]) }\end{array}$ & $\begin{array}{l}\text { 1) Consent (in written form) is required for any secondary use of health data. } \\
\text { 2) Health data must be kept confidential, integral, and available. } \\
\text { 3) Administrative safeguard, physical safeguard, and technical safeguard. } \\
\text { 4) Breach notification. } \\
\text { 5) HIPAA does not apply to de-identified health data. }\end{array}$ & $\begin{array}{l}\text { 1) Proper consent } \\
\text { 2) Confidential } \\
\text { 3) Integrity and necessary updates } \\
\text { 4) Available } \\
\text { 5) Transparency and fairness }\end{array}$ \\
\hline $\begin{array}{c}\text { EU } \\
(\text { GDPR [24]) }\end{array}$ & $\begin{array}{l}\text { 1) Lawfulness, transparency, and fairness. } \\
\text { 2) Minimum and limited data storage and collection, integrity check, and } \\
\text { up to date. } \\
\text { 3) The secure processing, data security including two-factor authentication } \\
\text { and end-to-end encryption, data protection by design and by default. } \\
\text { 4) Secure and compliance-friendly data transfer. } \\
\text { 5) Freely given, specific, informed and unambiguous consent, and data } \\
\text { subject rights to their data. } \\
\text { 6) GDPR applies to pseudonymized data if the data subject can be } \\
\text { identified by linking other additional available information. } \\
\text { 7) Breach notification }\end{array}$ & $\begin{array}{l}\text { 6) Reasonable, minimum, and limited } \\
\text { 7) Secure and privacy-preserving } \\
\text { data processing } \\
\text { 8) Secure and compliance-friendly } \\
\text { data transfer } \\
\text { 9) Proper administrative, physical } \\
\text { and technical safeguard } \\
\text { 10) Breach notification }\end{array}$ \\
\hline $\begin{array}{c}\text { Australia } \\
\text { (Privacy Act [45]) }\end{array}$ & $\begin{array}{l}\text { 1) Open and transparent management of personal information along with } \\
\text { anonymity and pseudonymity if not exempted. } \\
\text { 2) Reasonable and lawful collection of information only with consent and } \\
\text { notification. } \\
\text { 3) Prohibit disclosing PI, including marketing and cross-border, without } \\
\text { proper consent, and other tasks than the original purpose. } \\
\text { 4) Security and integrity. } \\
\text { 5) Access control and data correction. } \\
\text { 6) Breach notification. }\end{array}$ & \\
\hline
\end{tabular}

\subsection{Requirements due to ethics}

Ethical guidelines and framework are to ensure the responsibility of collection and analysis of $\mathrm{PH}$ data for any purpose. These enable us to decide the use of appropriate technology for social good. Recently, health (big) data analytic, which relies heavily on AI, has brought the ethical concerns of PH data more than ever on privacy, control, and data ownership. More precisely, ethical issues include the possibility of re-identification of users by linking, merging, data-mining, and re-using datasets in volume.

The study performed by an Italian company named Evodevo srl, with the support of the European Economic and Social Committee, explores the ethical dimension of Big data, which also includes PH data. It states the following ethical issues [49]:

1. Awareness: Lack of awareness can lead to unethical use of health data.

2. Control: To provide true ownership of the user's health data, users need to have control, including removal, of their private data provided to the service provider, including the data provided to other parties by the service provider.

3. Trust: Trust is required for the user's acceptance to provide personal data for a service (e.g., health advice).

4. Ownership: It is necessary to clearly state the ownership related to the data after processing the original user's data.

5. Surveillance and security: Unnecessary surveillance to limit the citizen's liberty is unethical.

6. Digital identity: The online profile of individuals due to his/her online activities can be used for discrimination.

7. Tailored reality: Focused and targeted service based on personalized information, including advice and advertisement, limits the user's exposure.

8. De-Anonymization: There are concerns related to linking the information from two or more sources to infer more information from the de-anonymized data.

9. Digital divide: Digital divide refer to an inability to use the new technologies (e.g., by senior citizens) for the services delivered through the new technologies. 
10. Privacy: Privacy is required to prevent the use of health data without consent.

The relevant requirements based on the issues mentioned above on PH data related to privacy, security, and trust are presented in the following:

1. Awareness, control, and ownership: It is required to practice informed use of data, provide user control over his/her data not only to the primary custodian, but also secondary custodians (which got the data from the primary custodian), and clear ownership criteria for the evolved data after processing the original user's data.

2. Trust: Ethical guarantees for the usage of data is necessary to gain trust from users.

3. Privacy: Privacy aspects are essential to preventing data usage without proper consent and approval, specifically, for secondary data usage.

4. Limiting the information linkage: It is required to preventing linkage of the data of one source to data from other sources to infer more information on the data subject other than the original intention. Thus, while releasing the health data to the public, proper consideration must be carried out such that there will be no or less possibility of extraction of sensitive information from the linkage.

National statement on ethical conduct in human research, Australia, states the following requirements on human data use in research [50]:

1. Ethics approval from the designated ethics committee: Ethics approval is the first step before conducting human research activities, including collection, store, and analysis of human-related data (such as PH data).

2. Consent: Consent should be taken voluntarily from the participants after providing adequate information about the proposed research and implications of the participation. Renegotiation of consent is required if the original terms change over time. Participants have opportunities to decline or withdraw consent. There are three types of consent in research, namely specific, which is limited to a particular project, extended and unspecified, which are given for the use of data in future research projects.

3. Address the following ethical issues related to the collection, use, and management of human data and information:

- Identifiability of information: It is required to de-anonymized the human data based on the requirements of the research, and proper care must be taken to reduce the likelihood of re-identification of individuals during collection, analysis, and storage of data.

- Data management: Proper access control and usage (e.g., analysis and re-use) are required if multiple researchers are collaborating on the same human data repository. Besides, other required measures for data management include physical, network, and system security, confidentiality agreement, and safe disposal.

- Secondary use of data or information: It is required to re-obtain the consent wherever applied. If unpractical to do so, then the usage must be ethically justified.

- Data sharing: It is required to follow the data management plan and ethical norms (including re-consenting if required and confidentiality agreement) while sharing data with other researchers.

- Dissemination of project outputs and outcomes: The dissemination of the inferences from the human data, including the outputs, need to be aligned with the ethical principles (e.g., the privacy of the participant).

4. Risk analysis and management: At various stages in research, there are risks associated with the privacy of human data and information. These risks need to be analyzed and managed properly at that stage.

Based on the American Medical Association code of medical ethics [51, we find the following requirements for the health data:

1. Privacy: A comprehensive privacy, including physical privacy, information privacy, decision privacy, and associational privacy, is required.

2. Confidentiality: For confidentiality, it is required to restrict disclosure of the data to third-party. If disclosure is needed for the benefits of the data subject, then only the minimum necessary information should be disseminated, considering re-obtaining consent if applicable. Any third party can have access only to de-identified information. Besides, the duty of confidentiality extends beyond the death of the data subject.

3. Medical records management: It is necessary to safeguarding and monitoring the confidentiality of the patient's personal information, proper access control mechanism to the data, its storage time limitation, and availability of the record if requested by a patient, or in point of care by a physician.

4. Breach notification: The patients must notify about the data breach if occurred.

In various other ethical guidelines, including the world medical association declaration of Helsinki - Ethical Principles for Medical Research involving Human Subjects [52], and a document by world health organizatin [48], primarily stress on the (i) privacy and confidentiality of personal information, and (ii) informed consent.

In overall, Table 2 and 3 provides the requirements for the legal and ethical compliances, respectively. 
Table 3: Summary of requirements due to ethical guidelines

\begin{tabular}{|c|c|c|}
\hline References & Requirements & Overall requirements \\
\hline \begin{tabular}{|l|} 
Evodevo srl 49$]$ \\
(supported by \\
EU Economic and Social Committee)
\end{tabular} & $\begin{array}{l}\text { 1) Awareness, Control and Ownership } \\
\text { 2) Trust (platform) } \\
\text { 3) Privacy } \\
\text { 4) Measures to limit the information linkage } \\
\end{array}$ & \multirow{4}{*}{$\begin{array}{l}\text { 1) Awareness } \\
\text { 2) Control } \\
\text { 3) Ownership } \\
\text { 4) Trust (platform) } \\
\text { 5) Privacy, security, and confidentiality } \\
\text { 6) Ethics approval in research projects } \\
\text { 7) Informed consent with the flexibility } \\
\text { to opt-out and transparency } \\
\text { 8) Proper de-anonymization to limit the } \\
\text { information linkage from various sources } \\
\text { 9) Proper data-sharing management } \\
\text { 10) Risk analysis and management } \\
\text { 11) Breach notification }\end{array}$} \\
\hline $\begin{array}{l}\text { National statement on ethical conduct } \\
\text { in human research, Australia [50] }\end{array}$ & $\begin{array}{l}\text { 1) Ethics approval } \\
\text { 2) Informed consent with an opportunity to decline or withdraw it } \\
\text { 3) Measures to limit the re-identification of individual } \\
\text { 4) Data management for security, confidentiality, and privacy } \\
\text { 5) Ethically justified or consented use of data for secondary purposes } \\
\text { 6) Ethical data-sharing management, including the outputs } \\
\text { 7) Risk analysis and management }\end{array}$ & \\
\hline $\begin{array}{l}\text { American Medical Association code } \\
\text { of medical ethics [51] }\end{array}$ & $\begin{array}{l}\text { 1) Privacy } \\
\text { 2) Confidentiality } \\
\text { 3) Medical records management, security, and privacy } \\
\text { 4) Breach notification }\end{array}$ & \\
\hline $\begin{array}{l}\text { World medical association declaration } \\
\text { of Helsinki [52], and } \\
\text { World health Organization [48] }\end{array}$ & $\begin{array}{l}\text { 1) Privacy and confidentiality } \\
\text { 2) Informed consent }\end{array}$ & \\
\hline
\end{tabular}

\subsection{Requirements due to health domain}

So far, we have discussed the requirements of the security, privacy, and trust from the data usage, collection, and storage perspective. Now, we present the domain-specific need that is not covered above. As the data are crucial for medical decision making, the data must be accurate, complete, and precise to avoid wrong decisions. An incorrect medical decision can harm the patients up to their death; thus, proper care must be taken. This results in the requirement of the $\mathrm{PH}$ data trustworthiness. In this paper, the requirements due to the issues related to the trustworthiness of health devices [53, which is also an essential issue in the medical domain, are out of scope. We explore only up to data trustworthiness.

The $\mathrm{PH}$ data trust is essential for the precision health that envisions to provide health care based on evidence derived from the data. We found a lower number of documents explicitly stating the requirements for $(\mathrm{PH})$ data trust. Based on our readings, including data trust [54], health data quality [55|56], and FAIR principle [57, the requirements are the following:

1. Standard format: Health data stored in a proper format across different organizations ensure ease of data processing, e.g., data matching, and data integration. Besides, the metadata and data should be easily human and machine searchable. There is a need for machine-readable metadata (e.g., proper indexing) maintained along with the data if the data search is automated.

2. Simple, clear and complete: Present data in a way such that further analysis and inference is consistent if it is processed at a different time or by different organizations.

3. Accurate, timely, and transparent: The health data should be accurate (e.g., correct data entry and updates). It should have adequate additional information to verify its credibility, including source information, data entry, and collection method. Transparency is also required to check the correctness of the data. The data should be recorded and processed on time to avoid possible errors and incompleteness.

\section{Major challenges in precision health data security, privacy and trust}

The following are the major challenges for PH data security, privacy and trust: (1) Health data security and privacy whilst computing, (2) consent management, (3) PH data trustworthiness, and (4) legal and ethical compliance.

\subsection{Health data security and privacy whilst computing:}

Security and privacy of data-at-rest are ensured by well-defined encryption methods such as Advanced Encryption Standard (AES) [58 8 Rivest-Shamir-Adleman (RSA) [59], Elliptic Curve Diffie-Hellman (ECDH) 60]. Besides,

\footnotetext{
${ }^{8}$ AES is quantum-safe as the AES-encrypted data can resist quantum attacks by increasing its key size. A cryptographic
} protocol is said to be Quantum-safe if it is well examined under all known quantum algorithm. 
query operations over the encrypted data (e.g., encrypted data-at-rest) without decryption is possible due to searchable encryption 61. Data-in-transit is protected by secure protocols such as Transport Layer Security (TLS) 62] and File Transfer Protocol Secure (FTPS) 63. On the other hand, protecting data security and privacy for data-inuse is a difficult task as it is associated with data computation, and whilst computation, the data usually requires decryption revealing itself to the computing platform. Moreover, the computing platform may not be a trusted platform. There are various evolving techniques to handle this issue, such as trusted platform, homomorphic encryption (computation over encrypted data), and multi-party computation. However, either they require a trusted vendor or they do not have product-ready protocols. These approaches are subjected to more research and development for its wider use.

Despite these challenges, there has been continuous progress in confidential computing, and related products and services are available in the market. Usually, these services are based on trusted platforms and memory encryption to isolate the data whilst computation, and provided by big technology companies, including Microsoft (Azure confidential computing) and Google (Google cloud confidential computing). However, recent studies show that these trusted platforms can be vulnerable to attacks, such as side-channel and timing. Refer to Section 4 for details in security and privacy for data-in-use.

\subsection{Consent management:}

Consent is mandatory for health data handling, including collection, analysis, and storage. It is an important tool to protect individual privacy, confidentiality, and autonomy. This is governed both by ethical guidelines and legislation. There are three types of consent, namely explicit, implicit and opt-out consent. In explicit consent, the purpose of collecting personal information, its use, handling, and disclosure of the information are presented with an option to agree or disagree. This type of consent is required for all aspects of clinical trials, including the retention of medical records. This is also called opt-in consent. It is used whilst handling the information. In implicit consent, consent is deemed in favor of both the data subject and collector. Most of the cases, this consent is obvious at the time of collection (e.g., a doctor taking blood samples of his patient for lab tests). In an opt-out consent, the participants are informed about the purpose of consent with an option to decline it. If it is not declined, then the consent is considered to be provided. A consent management solution for enterprises has been proposed by researchers at IBM [64]. This solution provides tools for modeling consent, a repository for storing it, and a data access management component to enforce consent and log the enforcement decisions.

The main problem related to consent arises whilst data sharing and data linkage. This is usually required in the data pre-processing phase of health data analytics, where data come from various sources (e.g., hospital, insurance company, and social media). There are two approaches for consent, namely static consent and dynamic consent. In static consent, the consent must be taken for all future usage of data at the time of data collection, and it is usually paper-based. It cannot address the issues that come with the change in environment and requirements with time, such as reusing the data for a different health project other than originally consented. In this regard, dynamic consent 65 66 is advantageous. Dynamic consent is an informed and personalized consent, where twoway communication is interfaced between the data subject and data custodian, and the subject can update and provide different kinds of consent. In addition, the subject can control their health data usage over time and revoke consent through the interface. Besides, the consent is traveled with the corresponding data when it is shared with other parties, and also, the participant can get the research results. However, dynamic consent has challenges, including higher implementation cost, consent revocation, and data deletion guarantee, and need to have patients with sufficient digital knowledge and time. Overall, how to automate the consent and manage it efficiently in the interest of legislation, patient's autonomy, cost, and data analytics is still an open problem.

The health data analytics require more health data, that means more participants and their consent, for better health care quality. Thus, it is equally important to explore the approaches that increase consent approvals. One possible way to do so is through trust (trustworthy system), as a study suggests that trust and privacy concerns are inversely proportional to each other 67 .

\subsection{PH data trustworthiness}

As health data is complex and diverse, checking and maintaining the trustworthiness of health data is a considerable challenge. In addition, the increasing size of health data (e.g., big data), distributed storage of health data (e.g., hospitals and pharmaceuticals) at different places, and a massive number of data sources (e.g., medical internet of things) add additional difficulties and complexities for checking the trustworthiness. Credible sources such as government agencies and reputed organizations are trustworthy data sources. They follow health data governance policies so that one can inspect their data via metadata and associated information. However, in the advent of the 
internet of medical things (IoMT), e.g., smartwatch monitoring heart rate, it is difficult to manage and maintain the reliability of health data, where data can be extracted from a faulty or improperly configured IoMT device.

\subsection{Legal and ethical compliance:}

Legal and ethical compliance is necessary while handling PH data. Otherwise, there may be a trust problem or hefty fine (e.g., $€ 20$ million or four percent of an organization's annual global revenue as stated by GDPR in EU) for the breach. To understand the privacy risks when conducting data processing (e.g., data analytic) and possible ways to reduce them for compliance, we present a summary of the guidelines presented in "Guide to data analytics and the Australian privacy principles" 68, as an example. Refer to Table 4 for details.

Table 4: Privacy risk factors and possible risk reducing steps

\begin{tabular}{|c|c|}
\hline Privacy risks & Possible risk reducing steps \\
\hline $\begin{array}{l}\text { Data may contain personal information, and it is sub- } \\
\text { jected to the Privacy Act. }\end{array}$ & Proper de-identification of data. \\
\hline No proper de-identification. & $\begin{array}{l}\text { Risk assessment to consider the likelihood of re-identification, and } \\
\text { implement risk mitigation techniques. }\end{array}$ \\
\hline $\begin{array}{l}\text { Privacy impact assessment (PIA) 69 is challenging for } \\
\text { big data. }\end{array}$ & PIA needs to be carried out. \\
\hline Using 'all the data' for 'unknown purposes'. & $\begin{array}{l}\text { Limit the collection and use of personal information to a reasonably } \\
\text { necessary level to perform legitimate functions. }\end{array}$ \\
\hline New personal information creation during analytics. & If not legally collected, then needs to be de-identified and destroyed. \\
\hline Information collected by third party included in analytics. & Follow the consent provided for secondary use of those information. \\
\hline People do not read privacy notices. & Customize the notices to make them easy, dynamic and user friendly. \\
\hline $\begin{array}{l}\text { Secondary use and disclosures of personal information are } \\
\text { common in data analytics. }\end{array}$ & $\begin{array}{l}\text { Check compatibility with the original purpose of collection or rely } \\
\text { on exceptions. Send privacy notices to inform individuals about the } \\
\text { particular use or disclosure. }\end{array}$ \\
\hline Impracticable to obtain individuals consent. & $\begin{array}{l}\text { Follow the law and guidelines (e.g., Australian Government National } \\
\text { health and Medical Research Council's guidelines } 70 \text { whilst handling } \\
\text { personal health information). }\end{array}$ \\
\hline Personal information disclosure to an overseas recipient. & Adopt extra diligence and follow law before disclosure. \\
\hline $\begin{array}{l}\text { Algorithmic biases in its decisions which are discrimina- } \\
\text { tory, erroneous and unjustified. }\end{array}$ & Ensure correctness of models and methods. \\
\hline $\begin{array}{l}\text { Information collected from third party may not be accu- } \\
\text { rate, complete and up-to-date. }\end{array}$ & $\begin{array}{l}\text { Take rigorous steps to ensure the data accuracy, correctness and up- } \\
\text { dates. }\end{array}$ \\
\hline Hacking risks. & $\begin{array}{l}\text { Take proper security and prevention measures (e.g., data encryption, } \\
\text { controlled access, and network security). }\end{array}$ \\
\hline
\end{tabular}

A compliance check is a difficult task, especially when data is collected from various sources, including third parties, and it is collected in a huge amount (usual case in big PH data). In addition, the law can be vague, and ethics are highly conceptual and abstract. It is unclear how to effectively and automatically check the compliance for data-in-use cases. However, by vigilant inspections, using proper platforms (e.g., privacy-by-design and privacyby-default), auditing (e.g., privacy impact assessment [69), using compliance analytic: 9 , and strictly following a compliance checklist in each data processing step starting from data collection to final output predictions, one can self-regulate the check and reduce the possible risks.

So far, we have explored the requirements due to regulations, ethics, and data trustworthiness for the PH data. We have identified that data security and privacy whilst computing is one of the main challenges. By considering its high impact in precision health, our survey is limited up to it in the remainder of this work; other major challenges are excluded. In this regard, we present the best available security and privacy-preserving techniques, including ML/AI techniques. These techniques ensure ethical and regulatory requirements while handling and using $\mathrm{PH}$ data in the healthcare domain.

\section{Techniques for $\mathrm{PH}$ data privacy and security}

Security and privacy of $\mathrm{PH}$ data or information is a mandatory requirement for health databases, including personal information (PI), worldwide due to legal provisions, financial reasons, and trust. In this section, we briefly discuss security and privacy-preserving techniques that are relevant to $\mathrm{PH}$ data. As one technology alone cannot provide a complete security and privacy solution, combinations of more than one are required.

${ }^{9}$ Compliance analytic calculates and prioritizes risk factors, and identifying highest risk transactions. These insights are used to manage the compliance risk. 


\subsection{Security and privacy for data-at-rest and data-in-transit}

Data security: There are four primary techniques for data security. These techniques are (1) cryptographic security, (2) blockchain-based security, (3) access control and security analysis, and (4) network security.

Cryptographic security: Cryptography [71] is an essential technique for data security against interception, tampering, and unauthorized reading. It deals with various data security aspects including authentication (checking and confirming the identity), integrity (ensuring only an authorized user makes modifications to the data), confidentiality (allowing only authorized recipients access the data), and non-repudiation (preventing the denial of earlier commitments or actions) 71. Data encryption plays a vital role in protecting sensitive information. However, its implementation is not extensive. According to Gemalto, in the first half of 2018, only $2.2 \%$ of the total data-breach incidents (worldwide) had data in encrypted form [72] (useless for the breacher). In the same report, health data breach accounts for $27 \%$ with the highest among all breach incidents by industry.

Encryption can be both software-based (e.g., Microsoft Windows BitLocker [73, VeraCrypt [74]) and hardwarebased (e.g., Seagate secure self-encrypting hard drives 75]). As the encryption and decryption are carried on by dedicated hardware components (not the main processor) in hardware-based encryption, it is faster than its software counterpart. Moreover, the encryption keys are stored locally (inside disk) in hardware encryption, which makes it more secure than software encryption, where keys can present in random access memory (RAM) locations whilst processing. Attacks such as cold boot attack can read keys present in RAM [76]. For disk storage devices, there are various storage encryption technologies, including full disk encryption, virtual disk encryption, volume encryption, and file/folder encryption, which provide different levels of securities [77. In a computing environment, disk encryption is not sufficient as information can be leaked from a processor or memory if it is not encrypted there. Thus memory encryption [78] and cryptoprocessors [79] are implemented along with disk encryption.

Blockchain-based security: Blockchain is a distributed public ledger that maintains a sequentially growing list of transactions or data in a chain of blocks. The information inside the blocks are immutable (no single party can delete it) and time-stamped. Blockchain enables data sharing without trusting the compute-nodes of a network, and a central node does not control it. Blockchain enables the security of networks and systems via data integrity. For example, Keyless Signature Infrastructure (KSI) blockchain [80] enables secure, scalable, digital, signature-based authentication for electronic data, machines, and PI. Also, KSI blockchain is quantum-safe. Estonia health care system [81] and Personal Care Record Platform, called MyPCR [82, use KSI blockchain to ensure data integrity and security in their system. Blockchain can be used for patient-driven healthcare interoperability. It can facilitate various aspects of interoperability, including digital access rules management, data aggregation, data availability and liquidity, patient identity, and immutability, though with some limitations, including handling the big $\mathrm{PH}$ data, privacy, security, and incentives considerations [83. To maintain the end-to-end confidentiality of genomic data queries, blockchain is used together with homomorphic encryption and secure multi-party computation [84]. Despite the ability to improve data security due to the data encryption on blockchain, there are possibilities of PI leakage from a public blockchain due to attacks, including linkage attacks [85. The scalability of blockchain, specifically, for the big PH data, is another primary concern.

Access control and security analysis: It is essential to secure physical devices (including desktop computers, laptops, and tablets) and infrastructures (including healthcare facilities, healthcare cloud servers, and data centers) that are holding sensitive private data. According to Verizon's 2018 data breach investigation report [86], $11 \%$ of the total data breaches involved physical actions, including theft of physical devices and paper documents. If an intruder gets access to those devices or infrastructures (premises), then he can obtain sensitive private data (i.e., data theft) and can damage the infrastructure or data (i.e., data loss). Other physical security risks include natural disasters, including fire, earthquake, and flood. A proper secure data backup system is necessary to recover the data when these risks occurred or system failure. Besides, access control mechanisms, which is a conventional approach to data security, regulate the users and their access to sensitive data. It performs identification authentication and authorization of users. Multi-factor authentication using passwords, bio-metric scans, cryptographic tokens, and RFID cards are standard mechanisms for access control. Besides, real-time security analytics device/software such as Intrusion Detection System (IDS) [87] and Intrusion Prevention System (IPS) [88] are essential security measures.

Network security: Network security maintains the security and privacy of data-in-transit. It is maintained through security protocols and standards such as Secure Socket Layer (SSL), Transport Layer Security (TLS), Secure HTTP, secure IP (IPsec), and Secure Shell (SSH). TLS and SSL provide transport-level security, IPsec provides networklevel protection, and Secure HTTP offers secure communication between a HTTP client and a server [89]. Protocols 
such as wired equivalent privacy (WEP) and Wi-Fi protected Access (WPA) protects wireless networks. Besides, an untrusted network such as the internet (a public network) consisted of security threats including computer virus, Trojan horse, adware, spyware, worm, and rootkit. A firewall, which is a network security system, and IPS (e.g., antivirus software) enable security in a local network that is connected to the untrusted network by monitoring and controlling all incoming and outgoing network traffic of the local network.

Data privacy: There are mainly three risks to data privacy, namely singling out, linkability, and inference [90. Singling out refers to the identifying individual/attribute/value in a dataset by isolating the records. In contrast, linkability refers to identifying an individual/attribute/value in a dataset by linking two or more other files related to the same individual/attribute/values. On the other side, inference refers to the possibility to identify the individual/attribute/values from the different individuals/attributes/values with a significant probability. The two primary techniques for data privacy are (1) anonymization and (2) pseudonymization.

Anonymization: Anonymization includes randomization and generalization 90. Randomization techniques modify the integrity of the data to avoid the active link between the data and the individual. On the other hand, the generalization technique generalizes or dilute the attributes of data subjects by changing the respective scale or order of magnitude. For example, writing region instead of the street, and a range of years rather than a specific year. Randomization is used against inference attacks, but not effective against singling out and link attacks. In contrast, generalization is effective against singling out but requires quantitative approaches to prevent linkability and inference. Randomization techniques 90] include noise addition (retain the overall distribution but hide individuals), permutation (shuffling the values of attributes in a table such that some of them are intentionally linked to different data subjects), and differential privacy (robust but there is a trade-off between the usability and anonymization, see Section 4.2). Generalization techniques [90] include aggregation, K-anonymity, and L-diversity/T-closeness. Aggregation and $\mathrm{K}$-anonymity protect against singling out by grouping them with, at least, $\mathrm{K}$ other individuals. On the other side, L-diversity is the extension of K-anonymity such that, in each equivalence class, every attribute has at least $\mathrm{L}$ different values to avoid inference attacks. And, T-closeness is the improved L-diversity such that equivalent classes resembling the initial distribution of attributes in the table are created to keep the data as close to the original one. Despite various techniques in anonymization, it is shown not sufficient for the privacy guarantee in a recent work [91].

Pseudonymization: Pseudonymisation [90] replaces one attribute in the dataset by another to reduce the linkability between the original identity of a data subject and the dataset. The techniques for pseudonymization include

- encryption with a secret key,

- hash function (a function that returns a fixed-size output from an input of any size and cannot be reversed),

- keyed-hash function with stored key (a particular hash function that uses a secret key as an additional input),

- deterministic encryption (a keyed-hash function with deletion of the key), and

- tokenization and masking (it replaces a part of data by a random or semi-random data, called token, which retains the format and data type of the replaced part of the data). For example, a dynamic data masking (MAGEN) by IBM [92] implements data masking to allow data sharing whilst safeguarding sensitive business data.

\subsection{Data security and privacy for data-in-use}

In this section, we introduce some important evolving data security and privacy-preserving techniques that are relevant to precision health, specifically for data-in-use cases. We discuss these techniques and their implementation in the healthcare domain. The summary of these techniques is presented in Table 5.

Trusted Execution Environment: Trusted Execution Environment (TEE) provides secure storage and isolation of sensitive computations from other processes, including operating systems, BIOS, and hypervisor. Moreover, it reduces the attack surface by isolation and cryptography, and thus increases the security of the processes running in TEE. TEE uses a hardware module or software module or both modules for the confidentiality and integrity of data and application code. Moreover, it has a mechanism for remote attestation that provides proof of trustworthiness to the users. TEE considers the threat model that includes all software attacks and physical attacks on the main memory and its non-volatile memory. There have been several works from industry and academia on providing TEE. These works include ARM TrustZone [93, Intel SGX [94, Trusted Platform Module [95, Intel TXT [96], AMD Security Technology [97, Sanctum [98, and Keystone Enclave 99]. For more insights into TEEs' environment and developments, we discuss some notable TEEs in the following. 
- ARM TrustZone [93: ARM TrustZone is a hardware level technology that enables the ARM processor system into two hardware-isolated zones, namely trusted zone and non-trusted zone. Both zones have their operating system and data. System modules like drivers and applications do not have direct access to the trusted zone. It is separated from the normal world operations, and thus from the attacks exploiting the normal resources. The trusted zone handles the sensitive operations and data that need to be secured. The secure context switching between the two zones is managed by special software called secure monitor in the case of Cortex-A processors, and a set of mechanisms (precisely three instructions, namely secure gateway, branch with exchange to nonsecure state, and branch with link and exchange to non-secure state) implemented into the core logic in the case of Cortex-M processors. Several academic research works and commercial products have used ARM TrustZone based TEEs [100]. Though the TrustZone is a key enabler for the development of trustworthy systems, it is vulnerable to various attacks, including those exploiting bugs in the TEE kernel, hardware exceptions, caches, and power management modules 100 .

- Intel SGX 94]: Intel Software Guard Extensions (SGX) are instruction-set architecture extensions that provide a trusted computing environment by leveraging trusted hardware. It uses secure containers, called enclave, for the protection and isolation of its contents (code and data) from other processes and other enclaves. The memory is encrypted with a key that is unique to each enclave. The enclaves are trusted components. Intel SGX provides a software attestation method that allows a remote client to authenticate the program executing inside an enclave. The implementation of Intel SGX in the real world for the development of various applications is made possible due to the availability of software development kits such as Intel Platform Developers Kit [101, Fortanix Enclave Development Platform [102] and Open Enclave SDK [103, and cryptographic library such as Intel SGX SSL library [104 dedicated for SGX. Unlike ARM TrustZone, Intel SGX has data sealing features and memory protection from physical attacks such as bus probing. However, one needs to trust the vendor fully. Besides, Intel SGX is vulnerable to side-channel attacks, including those exploiting page tables, caches, translation lookaside buffer, and DRAMs used by enclave programs [105. These attacks can be mitigated by using Compiler/SDK techniques and Microcode patch.

- Keystone Enclave [99]: Keystone Enclave is an open-source enclave for RISC-V processors. RSIC-V is an opensource hardware instruction set architecture (ISA). Keystone Enclave uses hardware capabilities in RISC-V to design a secure enclave. In contrast to the commercial and proprietary TEE environment (e.g., Intel SGX), open-source TEE environment provides transparency and inside details. This results in an experiment and research openly by academia and industry to address challenges in enclaves, including hardware vulnerabilities and side-channel attacks. Keystone security monitor, a special module running in machine mode (trusted mode), manages enclaves, Physical Memory Protection (PMP) entries, multi-core PMP synchronizations, and remote attestation. Memory and its buses are encrypted for the defense against physical attacks. Keystone Enclave has strong memory isolation enabled by using separate virtual memory management (other than that of Operating System) and ISA-enforced memory access management. It is a relatively new execution environment, and researchers are still working in its improvement and building software stacks, including toolchain and edge compilers.

It is still possible to emerge new attacks and defense mechanisms for TEEs. However, in all cases, the attackers should have privileged access or specific condition, which is not common in general.

TEEs and healthcare: Privacy and security of PI are of prime concern in the healthcare domain, and it is the most common use case of TEEs. Intel SGX is used to increase the trust and security of health data exchange in a Horizon 2020 project (a European Union Research and Innovation program) named KONFIDO [106. In KONFIDO, decryption, transformations, and encryption of patient summaries were carried out in the TEE provided by SGX. In another work, a privacy-preserving international collaboration framework for analyzing rare disease genetic data is introduced [107. This work leverages Intel SGX for trustworthy computations over distributed and encrypted genomics data. ARM TrustZone technology has been implemented to secure the medical internet of thing devices [108].

Homomorphic Encryption: Homomorphic Encryption (HE) allows the computations (arbitrary functions) over encrypted data without decryption. The computing environment would not be able to know the data and results, which both remain encrypted. Thus, HE enables secure computation on an untrusted computing platform. Depending upon the number of allowed operations on the encrypted data, there are three types of HE [109], which are as follows: 
1. Partially homomorphic encryption: Partially homomorphic encryption (PHE) allows only one type of operation on the encrypted data for an unlimited number of times. It supports either only addition or multiplication. Some examples of PHE schemes are RSA [59, GM [110], and KTX [111.

2. Somewhat homomorphic encryption: Somewhat homomorphic encryption (SWHE) allows more than one type of operation on the encrypted data but only up to a certain complexity and for a limited number of times. It supports both addition and multiplication, but the number of $\mathrm{HE}$ operations is limited because of the size of the ciphertext increase, and noise gets accumulated with each HE operation. Some examples of SWHE are Yao's Garbled circuit [112, SYY [113] on NC1 circuits, and IP on branching program [114].

3. Fully homomorphic encryption: Fully homomorphic encryption (FHE) allows any operations on encrypted data for an unlimited number of times. Gentry [115] first proposed a general framework for FHE. His scheme was based on ideal lattices. Further improvements in FHE schemes have been observed in several following works [109].

HE, especially FHE, plays a vital role in the privacy and security of PI. Its real-world implementation is challenging in general due to high computational requirements and overhead. Optimization has been done based on its use cases [116117, but it is still insufficient for a general case. The implementation of a fully functional FHE, whilst large data of different structures are input from multiple sources with different encrypting key, is an open problem. This type of environment is prevailing in precision health platform. Homomorphic encryption does not provide verifiable computing, so it should use other mechanisms for the purpose. For collaborative computations, HE can suffer from a collusion attack because all parties share the same public key, and the dishonest party can collude with the server [118.

HE and healthcare: As a privacy-enhancing technology, a lattice-based leveled 10 FHE scheme based on the Ring Learning With Errors (RLWE) problem is implemented for the protection of privacy and security of genomic data in i2b2 [120. The i2b2 is an open-source framework to enable sharing, integration, standardization, and analysis of clinical research data via collaborative efforts. In another work, (leveled) homomorphic encryption was implemented to conduct predictive analyses (e.g., logistic regression) on medical data 121] privately in a cloud service. In a recent work, it is used to achieve genome-wide association study, which compares genetic variants and single-nucleotide polymorphisms of genetic data, in a secure and private way [122].

Multiparty Computation: Multiparty Computation (MPC) enables distributed computations on encrypted data without decryption. It eliminates the need for a central trusted party for computations. Each data input is divided into two or more shares and distributes them among the multiple (distrustful) parties. All parties follow a protocol and jointly compute a function on their inputs without revealing their inputs to any other party. The final result is shared among them. In MPC, it is not required to store all data from different parties centrally, for which one needs to have a trusted third party. Yao 123 first introduced MPC in the early 1980s. It has been an important technique for privacy-preserving computations where data are distributed. The computational models of MPC include boolean, arithmetic, fixed/floating, and random access machine (RAM). For a secure MPC, it is proved that there is a bound on the number of parties being controlled by adversary or colluding [124. Homomorphic encryption [125], garbled circuits [123], linear secret sharing [126], and Oblivious Random Access Machine techniques [127/128] have been utilized for the construction of secure MPC protocols. MPC has also been used in secure neural network training [129].

Unlike HE, MPC has a low computational cost, but it has a considerable communication cost as its processes need to communicate encrypted data with each other across the network, and the communicating parties must remain online during joint computation. In MPC, the correctness of the computation (output) is ensured 130. Scalability is another issue with MPC. As the final result (after computation) may leak information about the inputs, MPC alone is not sufficient for privacy. Thus, a combination of MPC with other techniques such as differential privacy (see Section 4.2 or secure enclave is required for better privacy results.

MPC and healthcare: Healthcare is the best use case of MPC. In Scalable Oblivious Data Analytics (SODA) [131, a Horizon 2020 project, MPC is implemented as an underlying technology to preserve privacy whilst processing personal (health) Big Data from multiple distrusting parties (e.g., hospitals and insurance company). Refer to Section 6.1 for details. In another project, named San-shi, which is a secure computation system developed by Nippon Telegraph and Telephone Corporation (NTT), MPC is implemented for aggregation and statistical processing of

${ }^{10}$ In a "leveled" FHE scheme, the parameters of the scheme may depend on the depth of the circuits that the scheme can evaluate (but not on their size) 119. In simpler words, in a leveled FHE, functions are computed only up to a fixed complexity or level. There exists a conversion technique from a leveled FHE to (normal) FHE. 
Table 5: Summary of security and privacy preserving techniques for data-in-use

\begin{tabular}{|l|c|c|c|c|}
\hline & TEE & HE & MPC & DP \\
\hline Interactive & No & No & Yes & No \\
\hline Collaborative computing & Applicable & Applicable & Suitable & Suitable \\
\hline Implementation complexity (relative) & Low & High & High & Very Low \\
\hline Ensures correctness & No & No & Yes & No \\
\hline Computation speed (relative) & Fast & Slow & Slow & Fast \\
\hline Cryptographic technique & No & Yes & Yes & No \\
\hline Output data privacy & No & No & No & Yes \\
\hline Data protection & $\begin{array}{c}\text { Storage \& } \\
\text { Computing }\end{array}$ & $\begin{array}{c}\text { Storage \& } \\
\text { Computing }\end{array}$ & Computing & $\begin{array}{c}\text { Released } \\
\text { (output) } \\
\text { data }\end{array}$ \\
\hline Mathematical guarantee of privacy & No & No & No & Yes \\
\hline Network Communication & Low & Not required & High & Not required \\
\hline
\end{tabular}

confidential data whilst keeping the data encrypted 132133. Refer to Section 6.3 for details. In a different work, a privacy-preserving patient linkage technique is developed by using secure MPC based on Sharemind framework [134]. Sharemind [135] provides a secure infrastructure that hosts (usually) three nodes. Its framework is written in $\mathrm{C}++$. It processes privacy-preserving algorithms, and the security is achieved via secure MPC on additive secret sharing.

Differential Privacy: Differential Privacy (DP) provides the privacy of output data or results from computation or process such that the output data or results only reveal the permitted (which is usually negligible) amount of leakage of an individual input data. Dwork et al. [136] introduced DP in 2006. They provided an information-theoretic notion of privacy, called $\epsilon$-differential privacy, of a randomized algorithm. If the algorithm provides $\epsilon$-differential privacy for a small (near to zero) $\epsilon$, then adding or removing one data from its input data set does only nominal change to the outcome of the algorithm (the outcome lies within the multiplicative factor of $\exp (\epsilon)$ ) [136. In other words, a differentially private output ensures that any participant will not be affected adversely by allowing his/her data for analysis irrespective of studies and available datasets. The most common methods of realizing differentially private algorithms are Laplace mechanism [137] and exponential mechanism [138, where a random noise generated from Laplace distribution and a scaled symmetric exponential distribution is added to the output data to achieve DP, respectively. The added noise changes the output data nominally, and one can accurately learn the data, but it is sufficient enough to blur the individual input data (which cannot be learned precisely). More noise will make the data more private. Still, it reduces the quality of data and hence its utility. Consequently, a proper trade-off between privacy and utility is always desirable.

There are two types of DP, namely local and global differential privacy. In local DP, noise is added by each distributed participant to their input data before collection or computation, whereas in global DP, noise is added to the final output after computation. DP is an important privacy-preserving technique due to its properties. Some important features of DP [139] are the following:

- DP is immune to post-processing: Any post-processing of the output of a differentially private algorithm cannot make it less differentially private without additional information about the input (private) database.

- Composition of differentially private mechanisms is also differentially private: The composition of differentially private mechanisms are also differential private, where the total privacy losses are cumulative. Thus there can be a significant privacy loss when multiple differentially private computations are performed on an individual's data for a long time.

- The privacy guarantee drops linearly with the size of the group: The privacy guarantee deteriorates with the increase in the group size. Group privacy is different from composition privacy.

DP assumes that the initial data holders are always trusted, which may not be true in practice. It is a promising privacy-preserving technique but still has limitations, including difficulties in general computing of a global sensitivity that both guarantee privacy and acceptable level of noise and non-compact uncertainty (e.g., Laplace mechanism can change the original answer) [140.

Differential privacy and healthcare: DP has been extensively used in the healthcare domain, including releasing health data for research, and their analytic computations. As MPC alone does not guarantee privacy, a combination of DP and MPC is proposed as an underlying technology to preserve privacy whilst processing personal health 
data in SODA project 131. DP is also combined with an encryption technique. A combination of encryption with DP is used to guarantee the privacy of genomics data in a distributed clinical setup [141. In another work, a DP framework is integrated with the classical statistical hypothesis testing and applied to clinical data mining examples [142]. DP is implemented in distributed deep learning of two clinical data sets [143], where measurements of cumulative privacy loss are done by using Renyi differential privacy [144.

Before discussing the best available techniques and methods through a conceptual system model for the $\mathrm{PH}$ data security and privacy, we briefly introduce some important terms and approaches that we are using. Besides, we explore some relevant ML paradigms to healthcare, which considers PH data-in-use, in the following section.

\section{Methods of data storage, computing, and learning}

\subsection{Data storage and computing approaches}

Data storing methods: There are two common ways of storing PH data, namely centralized storage, and decentralized storage. In centralized storage, all the data from different sites (e.g., various hospitals) are collected and stored in one central server. It will be easier for computations if all data are available in one server. However, if the server failed or compromised, then this affects all data and systems associated with it. Besides, it is required to trust the server, and this increases the responsibility of the server to protect privacy and maintain the security of the stored information. Some examples of medical projects using centralized storage are 100,000 Genomes Project 145. and 23andMe [146]. In decentralized storage, the data are stored in multiple data servers. For example, each hospital can have its own data storage server. The data servers localize the risk of failure and attacks. However, it is relatively difficult for computations on distributed storage over centralized storage. Some examples of medical projects using decentralized storage are Global Alliance for Genomics and Health (GA4GH) [147, Swiss Personalized Health Network (SPHN) [148, and MedCo [149].

Computing approaches based on data accessibility: There are two types of computing approaches over the stored PH data ${ }^{11}[150$, based on data accessibility. These are (1) Data-to-modeler, and (2) Model-to-data.

Data-to-modeler: In the Data-to-modeler (DTM) approach, a data modeler has direct access to the data for the model development (training and validation) and hypothesis testing. This approach does not follow the norms of the privacy-by-design approach because the data modeler needs to be trusted, and data, which is sensitive in the healthcare domain, is directly accessed by the modeler. In some cases, this approach is infeasible. For example, different hospitals and insurance companies may not want to share their raw patient data directly with each other due to privacy concerns or competition or legal reasons. Data-to-modeler is a common approach whilst carrying out data analytics. As an example, for research purposes and discoveries, the anonymized health data sets are publicly released, such as health data provided by the Australian Government [151, HealthData.gov [152], and European Data Portal [153].

Model-to-data: In Model-to-data (MTD), the data is not directly accessible, and a modeler needs to submit their codes and models (obtained from their initial data) to the data contributor. In other words, the model moves to the data. Afterward, the models are trained and validated at the data contributors' server (or device) on the unseen actual data. The updated model or result is transmitted back to the modeler. The approach of MTD is privacy-by-design and data-centric. This approach seems promising in the case of health data analytics because it enables computing without seeing the patients' personal information. Federated learning (refer to Section 5.2 for details) uses the model-to-data approach, and it has been implemented in medical data analytics, including semantic segmentation models on multimodal brain scans [154] and prediction of mortality and hospital stay time [155].

Types of computing: There are three types of computing, namely (1) centralized computing, (2) distributed computing, and (3) decentralized computing.

In centralized computing, all the computations are carried out on one system/server. Use case examples of centralized computing are web application servers and mainframes. The centralized computing has major disadvantages, including single-point failure, scalability issues, and processing speed. On the other hand, in distributed computing, the computations are distributed to multiple systems or servers, but the process control and service requests are handled by one central system/server. Unlike centralized computing, this has no single point failure

$\overline{11}$ The storage can be centralized or decentralized. 
(increases reliability), scalable, and higher processing speed due to the parallelization of processing over multiple systems/servers. Hadoop [156], an open-source software, enables the distributed computing. Servers those running Hadoop, for example, Amazon EC2 [157, provide distributed computing services. In decentralized computing, both the computations and control of the processes are distributed among multiple systems/servers. Each computing node can process service requests. An example use case of decentralized computing is Blockchain [158. For example, iExec provides blockchain-based decentralized cloud computing services [159], and Golem delivers a decentralized marketplace for computing power (anyone can share their unused computing resources) [160. Decentralized computing includes all the benefits of distributed computing. In addition, it offers high availability and autonomy due to multiple service processing nodes. The complexity of the computing environment increases with the increase in the size of the network. For health-related data, including genetic data (usually Big Data) processing and analytics, either decentralized or distributed computing is more appropriate over centralized computing. This is because of high computational and storage requirements.

\subsection{Machine Learning paradigms and healthcare}

In this section, we present some relevant ML paradigms that are relevant to the healthcare domain. Table 6 provides a summary of these learning paradigms. These paradigms shed light on the $\mathrm{PH}$ data use whilst computing, and its transformation to knowledge in the form of ML models. This enables us to distinguish the privacy-preserving ML techniques required for $\mathrm{PH}$ data.

Transfer Learning: Transfer learning [161 leverages pre-trained ML models by reusing them for new related problems. In other words, it transfers the knowledge gained from one problem (in one domain) to the related target problem (in another but similar domain). This transfer of knowledge improves learning in the target problem. The transfer learning approach is useful to train a model even if the data is insufficient because the model is pre-trained with sufficient data on the related problem. It is used in Deep Learning (which requires a large amount of data to model its neural networks) [162], Natural Language Processing (enables machines to understand, process, and manipulate human language) and Computer Vision (allows machines to process images to identify objects). This learning methodology is inappropriate if the problems are not sufficiently related. Transfer learning in deep learning suffers from catastrophic forgetting, meaning that the network forgets its previously learned information once it learns the new information [163. Various works, including learning without forgetting [164], progressive neural networks [165, and elastic weight consolidation [166, have been proposed to address the catastrophic forgetting problem.

Transfer Learning and healthcare: Transfer learning has been extensively used in medical image analysis [167 168. The learned codebook from 15 million images collected from ImageNet [169] is used in Otitis Media (a group of inflammatory diseases of the middle ear) images with a detection accuracy of $88.9 \%$ [167. In another work, GoogLeNet [170] and AlexNet [171] models are reused and shown to be useful for thoracoabdominal lymph node detection and interstitial lung disease classification problems [168]. A trained model on the ImageNet [169] database has been reused to train fundus images for the detection of Glaucomatous Optic Neuropathy with higher performance and faster convergence [172].

Multi-task Learning: Multi-task learning is an approach to inductive transfer that improves generalization by using the domain information contained in the training signals of related tasks as an inductive bias [173. It adopts the concept of collective transfer learning, where one task helps another related task to learn better. In other words, multi-task learning aims to improve the overall performance of multiple associated tasks [174]. Unlike transfer learning, which focuses on solving one task at a time, multi-task learning solves the various tasks at one time by leveraging the similarity and differences across tasks. As all tasks are learned at the same time, the gained knowledge is available to all tasks. This process of learning is also called parallel transfer. The order in which the tasks are trained makes a difference in transfer learning; on the other hand, in multi-task learning, due to the parallel transfer, it does not make any difference. Thus there is no need to define a training sequence in multi-task learning [173].

Multi-task Learning and healthcare: Multitask learning has been applied extensively in the healthcare domain. It is used for drug discovery, where models were trained on 259 datasets, including PubChem BioAssay, datasets designed to predict interactions among proteins and small molecules, and database designed to avoid common pitfalls in virtual screening [175]. It is shown to have a significant performance in terms of accuracy to baseline ML methods such as logistic regression and random forest [175. In another work, a multi-task learning formulation 
(temporal group lasso multi-task regression) for predicting the Alzheimer disease progression is proposed [176. The disease progression is measured by cognitive scores based on baseline measurement, and the effectiveness of the proposed formulation is evaluated by experimental studies on the Alzheimer disease neuroimaging initiative database. In another work, conditions of mental health based on social media text are modeled as tasks in multitask learning. This work shows that the model predicts potential suicide attempts with an accuracy of above $80 \%$ in limited training data conditions [177]. Besides, applications of multi-task learning include clinical prediction [178, decompensation prediction (predicting whether the patient's health will deteriorate in the next 24 hours) [179], and ECG data analysis [180].

Continuous Learning: Continuous learning is a type of ML method that offers to learn from the newly available data over time such that it retains its previously gained knowledge and selectively transfers that knowledge to learn a new task. This way, the model gets benefited from the newly available data without learning from scratch each time whenever data is available. Continuous learning is suitable for the cases where data gradually available over time, i.e., data streaming (e.g., data coming from health monitoring devices), or the size of data is big enough to be out of system's memory, i.e., big data (e.g., human genome information). This learning is usually done for better performance (e.g., accuracy). There are various terms including incremental learning [181|182], online learning [183], and lifelong learning [184] in literature, and we refer all to the continuous learning as these learning methods learn continuously with time. Continuous learning uses Transfer learning as its integral part. However, transfer learning is not concerned with continuous learning and knowledge retention. The disadvantage of continuous learning includes continuous computation and prone to catastrophic forgetting.

Continuous Learning and healthcare: Incremental learning is used to build a medical image segmentation framework [185. This framework is applied to lung boundary delineation in High Resolution Computed Tomography scans, and experimental results show that it outperforms the fixed or non-adaptive algorithms. In another work, incremental learning algorithms, called $i^{+}$Learning and $i^{+}$LRA, are introduced based on decision tree learning methodology [186. On various medical data sets from the UCI repository [187, these algorithms are shown to be providing better classification accuracy than the other learning algorithms, including incremental tree induction [188] algorithms.

Ensemble Learning: Ensemble Learning implements several (similar or different) learning algorithms (models) to solve the same problem, and then combine the outputs from each algorithm (model) to get a better final result in terms of predictions or classification [183|189] of the problem. Usually, the final result is decided based on the voting (e.g., majority voting, weighted voting, and Bayesian voting) and statistical process (e.g., averaging and bootstrap aggregating). The voting and statistical methods are usually done for classification and regression, respectively. Support Vector Machine is also used in the voting process [190]. We can implement the ensemble learning in two ways. In the first way, different learning algorithms are trained over the same dataset, whereas in a second way, the dataset is split into disjoint subsets, then the same or different learning algorithms are trained over each subset of the dataset. Ensemble learning can be used in incremental learning.

Ensemble Learning and healthcare: Ensemble learning has been applied in the medical diagnosis of Alzheimer disease based on Magnetic Resonance Imaging datasets 191. Experiments are performed on 416 subjects of the OASIS database implementing Extreme Learning Machine, Bootstrapped Dendritic Computing (BDC), Hybrid Extreme Random Forest, and Random Forest with BDC scoring the highest of 80.8\% accuracy among others [191. In another work, two adaptive distributed privacy-preserving algorithms based on a distributed ensemble strategy are proposed for the health care domain. The algorithms were tested on a Type-2 diabetic electronic health record dataset and showed that the ensemble learning over distributed datasets is better than the learning on each dataset separately 192. In a different work, an ensemble learning, called asBagging_FSS, is proposed [193]. The asBagging_FSS algorithm is shown to have a good performance (i.e., accuracy) on high dimensional and imbalanced biomedicine datasets, including cancer DNA microarray and cancer protein mass spectrometry datasets [193].

No-peek Learning: No-peek learning refers to the distributed deep learning techniques that do not require the sharing of raw data available at distributed sources. It is based on collaborative learning and includes federated learning, split learning, and stochastic gradient descent (SGD) based collaborative learning. 
Table 6: Summary of machine learning paradigms

\begin{tabular}{|c|c|c|c|c|c|}
\hline & Transfer & Multi-task & Continuous & Ensemble & No-peek \\
\hline Model reuse/share & Yes & Yes & Yes & No & No \\
\hline Insufficient data & Suitable & Suitable & $\begin{array}{c}\text { Suitable provided that } \\
\text { data is available with time }\end{array}$ & No & $\begin{array}{c}\text { Suitable } \\
\text { is distributed and collective } \\
\text { data is sufficient }\end{array}$ \\
\hline Continuous learning & No & No & Yes & No & No \\
\hline $\begin{array}{c}\text { Multiple (different) models } \\
\text { Model-to-data and } \\
\text { distributed computing }\end{array}$ & No & No & No & No & Yes \\
\hline
\end{tabular}

Federated Learning: Federated learning (FL) is a collaborative ML technique 194195/196 197 developed by Google for training models on the training data that are distributed on mobile devices. It moves the computing to the edge devices. It preserves the data privacy at each participating edge devices. In the traditional ML approach, the training data are usually available in one central server (or datacenter), and the training takes place in that server, which is generally a trusted platform and can see data whilst computing. In contrast, FL approach preserves the privacy of data available at the edge devices by performing collaborative learning that never requires the data in the distributed edge devices to be collected out of those devices. More precisely, firstly, the coordinating server trains a global model based on its available data, then it sends the model to a set of devices. Secondly, each of those devices trains the model and computes an update based on the local training data. Thirdly, the updated model parameters are sent to the coordinating server, which aggregates those updates securely by using secure aggregation protocols 198 . without learning individual inputs from the devices. Finally, the coordinating server updates its global model based on the new aggregate model parameters. This process is repeated until the model parameters converge. Thus there is a communication cost in FL. TensorFlow Federated framework [199] and PySyft [200] library are enabling the implementation of FL. Besides, FL is an active field of research [201].

Challenges in FL includes communication cost between the edge devices and the coordinating server, availability of edge devices, massive distribution of edge devices, and privacy-preserving aggregation at the coordinating server. Besides, attacks such as inference attack (leaking information from the aggregated updated model parameters) 202|203|204, and poisoning attack (poison the training data to compromise the global model) 205] are possible in FL environment. It is not guaranteed that the updated model parameters to the coordinating server would not reveal sensitive information about the user, so FL requires other privacy-preserving techniques, including Differential Privacy (see Section 4.2) for robust privacy protections.

Federated Learning and healthcare: FL has a vast application in health data analytics. It makes possible to a distributed ML over patient health records privately and without requiring those data out of the hospital's secure data center or user's personal devices. Intel has demonstrated the first real-world medical use case of FL. Intel uses FL to train a deep learning model without sharing medical imaging data among collaborating clinical institutions. They achieve $99 \%$ of the model performance of the same model trained with the traditional (i.e., centralized) data-sharing method 154. In another work, a novel FL model for an optimization of the performance of predicting mortality and hospital stay time is proposed. The experiments in this work show that the proposed model has predictive accuracy close to that of centralized learning [155. In separate work, FL is used to predict hospitalization for patients with heart diseases using their electronic health records resided in different hospitals or sources [206]. Recently, FL techniques are implemented to analyze the distributed electronic medical records (including non-IID ICU patient data) for an efficient prediction of mortality and hospital stay time 207. Federated learning is also employed on brain tumor segmentation data with a comparable segmentation performance to the centralized system [208.

Split Learning: Split learning, also called split neural network (SplitNN), is a type of distributed deep learning [209210/211212]. Lile FL, SplitNN is useful specially when raw data sharing among the data holders/sources is not possible due to resource limitations or privacy and legal reasons. In this learning, firstly, each client trains a neural network up to a particular layer, called cut layer, and transmits the output of that layer, called activation or smashed data, to the server. After receiving the activation from the client, the rest of the layers of the network is trained by the server and completes the forward propagation. Secondly, the server computes the loss based on the true and the predicted labels, and backpropagate the loss to calculate the correction in the form of gradients until the cut layer. Then the gradient of the cut layer is transmitted back to the client. After receiving the gradients, the client continues the backpropagation of the network on its side. This way of forward and backward propagations 
continue until the network is trained. The client-side computational and communication requirements are reduced in SplitNN because the client only processes a part of the network, and the data to be transmitted to the server is the activation of the cut layer. The activation is relatively smaller in size compared to the raw data. As the model is split and trained in client and server, SplitNN also protects the trained model's detailed architecture and parameters. This kind of protection is not available in FL. Besides, this technique is similar to FL, as both are distributed ML techniques. SplitNN is shown to have better accuracies and lower computational requirements than FL and large batch synchronous SGD over CIFAR 10 and CIFAR 100 datasets using VGG and Resnet-50 architectures for 100 and 500 clients based setups, respectively [209]. However, FL has lesser communication bandwidth than others with smaller (i.e., 100) number of clients [209]. It is still an open research problem to make the algorithms and decisions made by deep learning techniques like SplitNN (whose processes are usually blurred to the outside world) explainable as demanded by regulations such as GDPR (EU). Moreover, the explainability factor is associated with the trust of the system, and it is crucial in the health domain.

Split Learning and healthcare: Configurations of SplitNN, including simple vanilla configuration, U-shaped configuration for split learning without label sharing, and vertically partitioned data for split learning, are proposed for various practical health settings 209. SplitNN is improved to reduce the information leakage at the cut layer of the neural network. The improved SplitNN is called NoPeekNN, and it is tested with a dataset of colorectal histology images without any data augmentation [211. Recently, SplitNN is studied to analyze the diabetic retinopathy dataset with Resnet-34 and chest X-ray dataset with DenseNet121 in a distributed setting with up to 50 clients. Its accuracy is shown to be better than a non-collaborative setting [213]. In recent works, SplitNN is used together with differential privacy to demonstrate machine learning with privacy on ECG dataset [214], and with IoT gateways [215].

Stochastic Gradient Descent: Stochastic Gradient Descent (SGD) based collaborative learning works in similar essence to FL. The difference is that the updates (local updates and global updates) in SGD based collaborative learning are based on one batch of training data, whereas, in FL, a client trains the model for some local epochs before updating it to the server. In SGD based collaborative learning, the parallelization of the updates is done through either model parallelism (a part of the model is evaluated in one machine) or data parallelism (model parameters are computed over a batch of data available in one machine). It includes Distributed Selective SGD [216], Large Minibatch SGD [217, and Distributed Synchronous SGD with backup workers 218210.

In Distributed Selective SGD, each client chooses a fraction of parameters (of its local model) to be updated at each round and share it asynchronously with other clients through a parameter server. On the other hand, Large Minibatch SGD considers synchronous SGD by dividing SGD mini-batches over a pool of parallel workers, and gradient aggregation. The updates are done synchronously, meaning updates on model parameters take place only after receiving all updates from all machines. As the straggling clients slow down the whole process, synchronous optimization with backup workers (computing nodes) in distributed synchronous SGD is proposed [218].

\section{Related health projects, their privacy and security measures}

In this section, we present some relevant projects addressing security and privacy in the healthcare domain. These projects include Horizon2020 funded projects, Swiss personalized health network projects, and Nippon Telegraph and Telephone Corporation project. Table 7 presents a summary of these projects.

\subsection{Horizon2020}

Horizon2020 is the biggest EU research and innovation program, with funding from 2014 to 2020 [219]. There are several health-related projects under Horizon2020, including My Health My Data, Scalable Oblivious Data Analytics, and KONFIDO. We present the relevant projects in the following.

My Health My Data: The project My Health My Data (MHMD) 220221222 spans from Nov. 2016 to Oct. 2019. The main aim of this project is to introduce a new way of sharing medical information and empowering their primary owner, the patient, using encryption and blockchain technologies. The key proposed elements include the following:

- Blockchain: Blockchain is used for ensuring the lawfulness and legitimacy of data exchange, keeping track of data access, and improving data integrity. MHMD implements blockchain by identifying its key features in a 
healthcare context, including high transaction rates, low network latency, low energy consumption, scalability, and robust privacy features. More precisely, a hyper ledger is implemented because the healthcare ecosystem requires a federated blockchain with a consensus mechanism such as proof of stake or practical Byzantine fault tolerance.

- Smart contracts: Smart contracts are self-executing protocols that can facilitate, verify, and enforce the performance of the contract. MHMD uses it to automate peer-to-peer transactions under user-defined data access conditions. The MHMD smart contract includes data registration, data access request, privacy-preserving for data transactions, study updates, and an indication of available data matching the study request.

- Personal Data Accounts: Personal Data Accounts (PDA) is a personal data storage clouds that enable individuals to access their data from any sources including wearable, clinical data, and social media, and use them for personal use. This service is provided through digi.me's secure personal data library and consent access process platform in this project.

- Dynamic consent: Dynamic consent [66] enables the possibility for individuals to provide different types of consent according to distinct potential data uses, taking control over the data access by others and its purpose. In MHMD, it is envisioned to use smart contracts for the implementation of dynamic consent.

- Secure computation and privacy-preserving mechanisms: MHMD implements both semantic multi-party computation and partial homomorphic encryption techniques to ensure the secure computation of sensitive information. For data privacy of the published datasets are provided through anonymization techniques such as k-anonymity and differential privacy.

Scalable Oblivious Data Analytics: The project Scalable Oblivious Data Analytics (SODA) 223|224|225|226|227. spans from Jan. 2017 to Dec. 2019. The main aim of this project is to enable practical privacy-preserving analytics on (big) data from multiple datasets with healthcare as the first use case. The fundamental techniques include multi-party computation techniques and differential privacy. In their work, they show that the MPC alone does not guarantee privacy and anonymity because the information leakage can occur from the output. Thus, they combine MPC and differential privacy for big data analytics by using MPC framework MPyC, an MPC tool available as a python package designed to support secure $m$-party computation tolerating a dishonest minority of up to $t$ passively corrupt parties 228, in three-party mode. For the implementation of MPC, SODA provides improvements and expansion of the FRESCO framework 12 229], and use them to implement MPC. The upgrades include application interface, SPDZ preprocessing, network improvements, the addition of TinyTables protocol with semihonest security, and SPDZ2K protocol [230, new library functionality (e.g., comparison protocol and aggregating functionality based on an encryption technique), and MPC based ML. As a part of the project, an MPC-enabled query compiler, called conclave, is proposed. Conclave addresses the implementation issue of MPC regarding the domain-related knowledge and scalability issue with big data sizes. More precisely, conclave considers three parties and allows relational queries to be processed among the parties by turning the queries into a combination of local processing steps and secure MPC steps. Other works towards the realization of general-purpose MPC includes efficient secure computations in rings, scaling up MPC to many parties (introduce short keys for secure computation), communication-efficient honest-majority MPC from batch-wise multiplication verification, two-party computation from oblivious linear function evaluation, differentially private logistic regression, and controlling leakage in MPC on non-integer types.

KONFIDO: The project KONFIDO [231|232|233] spans from Nov. 2016 to Oct. 2019. The main aim of the project is to advance the eHealth technologies for data preservation, data access and modification, data exchange, and interoperability and compliance primarily across European countries. KONFIDO proposes security components for OpenNCP, which is a software implementation of smart open services for European patients (i.e., epSOS) for seamless health data exchange across borders. KONFIDO proposes the use of the following in its system:

- Cryptographic techniques for the security (integration of Intel SGX and fully homomorphic encryption with OpenNCP, respectively),

- physical unclonable functions (based on photonic technology) and commercial off-the-shelf CPU technology for a secure data exchange, homomorphic encryption or secure enclaves for secure data processing,

- extension of the selected security information and event management (SIEM) to include multiple independent monitoring nodes,

$\overline{12}$ A FRESCO is a hybrid-mode MPC (software) framework offering a high degree of modularity, parallelization and preprocessing, and it is available as a Java library. 
- KONFIDO Secure Identity Across Borders Linked (STORK) 234 compliant eID support for authentication of the operators (developed an eIDAS-compliant eID for OpenNCP), and

- blockchain-based log management system for traceability and liability (e.g., informed consent) of health data sharing and data access permission handling.

\subsection{Swiss personalized health network}

Swiss personalized health network (SPHN) is a national initiative designed to promote the development of personalized medicine and personalized health in Switzerland, and this project spans from 2017 to 2020 [148]. It envisions for the harmonization of the information system, and data types between the participant hospitals and research institute, and facilitate the nationwide health data exchange for health research. There are several projects under SPHN. We present one project that is related to the security and privacy of health data in the following.

MedCo: The project MedCo [149]141] aims to develop a workable system that provides security and privacy to the sensitive medical data available for research. This system enables investigators to share their medical data in compliance with regulations. MedCo enables the data sharing through a hybrid or "somewhat" decentralized approach that overcomes the limitations of a centralized system (e.g., single-point failure), and a fully-decentralized system (e.g., need of operational resources and costs on the clinical sites). MedCo distributes trust among local Storage and Processing Units (SPU) and shared SPU. Local SPU is dedicated to the local site, whereas the shared SPUs provides services to the multiple sites (who do not have sufficient resources to maintain its local site). MedCo is built on top of two existing open-source technologies for medical data exploration, viz., i2b2 [235], and SHRINE [236]. It is tested in a simulated federation of three sites by focusing on a clinical-oncology case with tumor DNA data from The Cancer Genome Atlas. MedCo proposes to use privacy-preserving techniques, including homomorphic encryption, secure distributed protocols, blockchains, and differential privacy. MedCo implements the additively homomorphic encryption (partially homomorphic encryption that satisfies only the addition of ciphertexts where the key is generated collectively). This encryption ensures end-to-end protection by allowing queries and processing on the encrypted data (without decryption). Also, a set of SPUs (more than one SPU) are required for the decryption of the encrypted data, so the system is robust till all the SPUs in the set are not compromised.

\subsection{Secure Computational System San-Shi 132 133 237}

San-Shi is a project of Nippon Telegraph and Telephone Corporation (NTT). It enables aggregation and statistical processing with the functional performance of encrypted data. The data include multi-facility clinical research data. Basic operations include (1) the data operation, e.g., table join (without leaking the join key), (2) filtering by conditions, e.g., NULL filter and data filter with date/strings comparison, (3) aggregation, e.g., frequency table (cross-tabulation and histogram) and quantity table, and (4) statistics, e.g., total sum and variance, t-test, and Kaplan-Meier method. San-Shi enables data integration among different companies and cross-analysis without releasing the individually held data. The key technologies include encryption techniques and multiparty computation based on the secret sharing scheme. San-Shi's system consists of up to four servers, where a client protects data as shares, registers them in each server, requests computation to each server, and collects the results from the servers. San-Shi is tested on the secure and private analysis of genomics data.

\section{Discussion}

So far, we have investigated requirements and available techniques for PH data security and privacy. Now in this section, we list out the best available techniques through an illustration of a conceptual system model.

\subsection{A model for computation on distributed PH data}

In a practical setting, health data are decentralized and reside in various locations and institutions, including hospitals, pharmacies, personal mobile devices, research centers, and social media, as shown in Figure 3 . The first challenge is how to ensure the requirements listed in Section 2. In this regard, we present three polices and two types of consent. Policies include consent policies, ethics policies and privacy policies, and consent includes dynamic consent, and smart consent.

Consent policies ensure the fair use of data and check against PH data's metadata. Ethics policies ensure the requirements related to algorithms and analytics, and check against the algorithm's metadata. Privacy policies 
ensure the overall privacy of the system's environment, including compute, storage and communication, and check against the algorithm's and data's metadata. These policies are the first to be checked before ML meets data (i.e., computation). The right figure in Figure 2 illustrates the execution steps. Overall, each program code (for PH data processing) and $\mathrm{PH}$ data has metadata and policy. The policy of one is cross-checked with the metadata of the other, e.g., metadata about data is checked with ethics policy attached to the code, and metadata of the code is checked against consent policy attached to the data. Both metadata are checked against privacy policy as well. These checks are round 1 checks. In round 2 , if all checks return true, then code and data can meet for execution. This type of computation assumes the use of cryptography and TEEs.

Regarding consent, smart contracts provide a clear description of data handling and its purpose, secure storage of consent, access to withdraw the consent at any time and to renew the consent periodically. On top of it, dynamic consent enables us to have different consents for different uses of the data. How to execute these consent policies and perform their management in a practical healthcare setting is still subjected to further research.

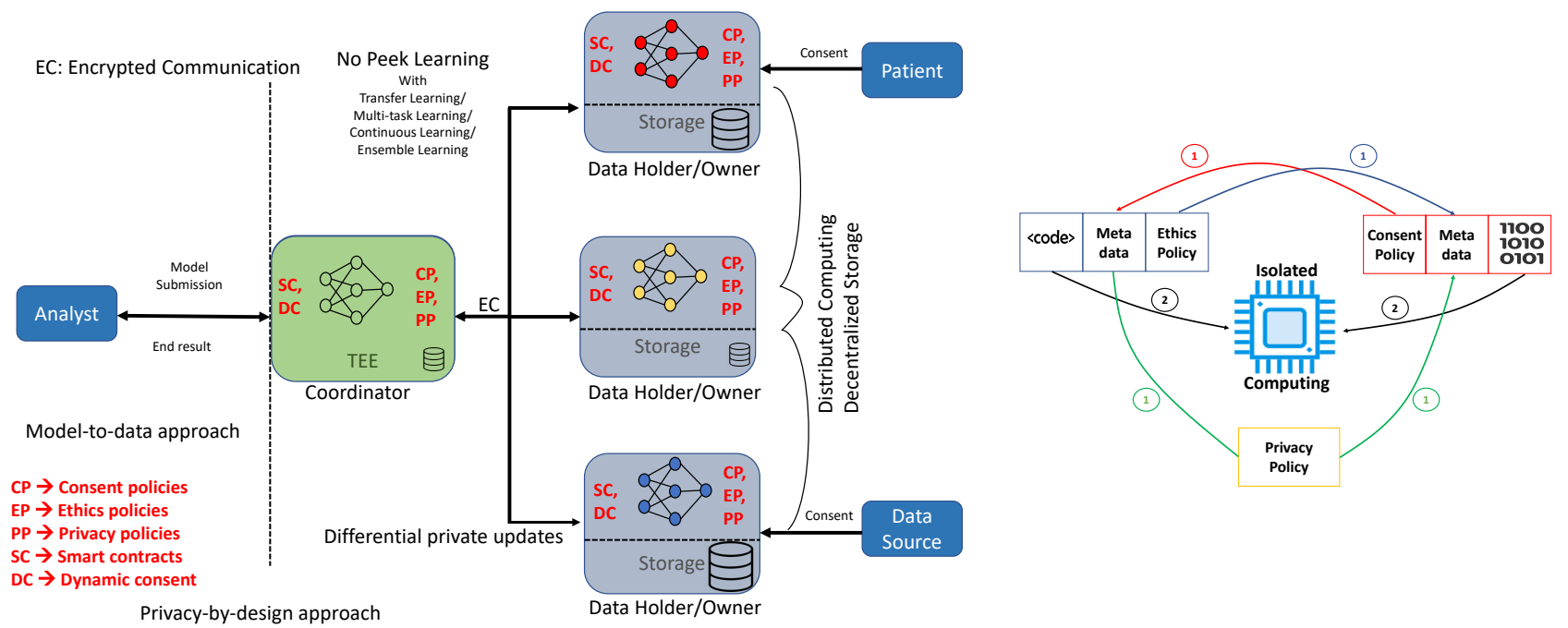

Fig. 2: A model for precision health platform (left figure), and execution of different policies (right figure).

The other challenge includes health data sharing and integration. It is remained as a constraint to health data analytics because of privacy reasons or reluctant to share the data due to the competition. Thus, the health data have remained in silos. In this regard, we choose an alternative approach to direct data sharing and integration. By considering the methods described in Section 4.2 and 5.2 , we envision that No-peek learning, specifically, federated learning, split learning and their variant splitfed learning [238, make the PH data analytics possible without sharing the data (meaning that the raw PH data never leave their sources such as hospital and pharmacy). Besides, it is not required to rely on other devices or services to delete the participants' sensitive PI once they decide to opt-out from the system (in this regard, it eases the consent management). Thus the No-peek learning maximizes the user's value and increases trust. The model-to-data method, such as No-peek learning, is a privacy-by-design, and it enables distributed computing to handle the distributed PH data. Consequently, medical innovations and development are possible due to the breakage of the data silos (e.g., distributed medical records) in the medical domain. Single security or privacy-preserving technique is not sufficient to provide all requirements, so the presented system model integrates trusted execution environment and differential privacy to the No-peek learning to offer a complete package that ensures the privacy, security, and trust of the system. Other techniques such as homomorphic encryption and multiparty computations can be implemented in the proposed system model, but we exclude them by examining their high computational requirements and their initial stage of technology readiness for a general scenario. Health data security and privacy for data-at-rest and data-in-transit are ensured through the existing methods described in Section 4.1 Figure 2 depicts the overview of the platform, and Table 7 provides a comparative summary with other projects.

Now, as an example (refer to Figure 3), we illustrate the system model considering federated learning, differential privacy, and Intel SGX. In this system model, data stored in all end devices and all the communication channels are encrypted at all times, and the authorized recipient having a proper key can only decrypt the data/information. 


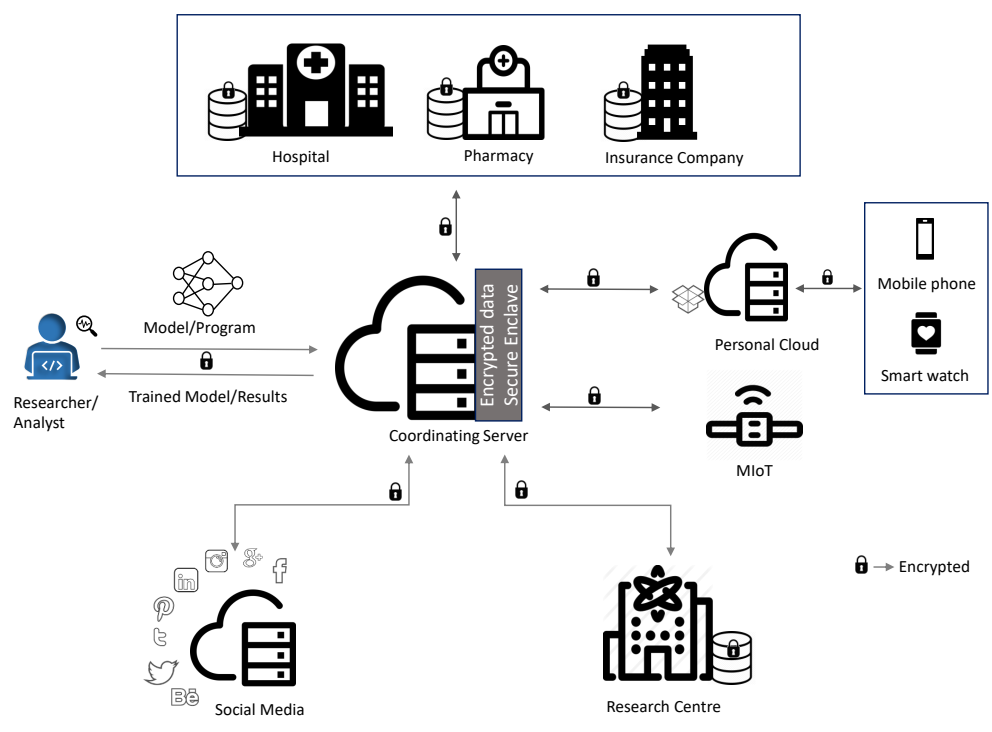

Fig. 3: An example case of the precision health platform

Table 7: Summary of the related health projects and their techniques

\begin{tabular}{|c|c|c|c|c|c|c|}
\hline Techniques & MHMD & SODA & KONFIDO & MedCo & San-Shi & Presented model \\
\hline Model-to-data & $\boldsymbol{x}$ & $\boldsymbol{x}$ & $\boldsymbol{x}$ & $\boldsymbol{x}$ & $\boldsymbol{x}$ & $\checkmark$ \\
\hline Blockchain & $\checkmark$ & $\boldsymbol{x}$ & $\checkmark$ & $\checkmark$ & $\boldsymbol{x}$ & Possible \\
\hline Homomorphic encryption & $\checkmark$ & $\boldsymbol{x}$ & $\checkmark$ & $\checkmark$ & $\boldsymbol{x}$ & $\boldsymbol{x}$ \\
\hline Multi-party computation & $\checkmark$ & $\checkmark$ & $\boldsymbol{x}$ & $\boldsymbol{x}$ & $\checkmark$ & Possible \\
\hline Differential privacy & $\checkmark$ & $\checkmark$ & $\boldsymbol{x}$ & $\checkmark$ & $\boldsymbol{x}$ & $\checkmark$ \\
\hline TEE & $\boldsymbol{x}$ & $\boldsymbol{x}$ & $\checkmark$ (Intel SGX) & $\boldsymbol{x}$ & $\boldsymbol{x}$ & $\checkmark$ \\
\hline Policies enforcer (trustworthy checks) & $\checkmark$ & $\boldsymbol{x}$ & $\checkmark$ (Blockchain based) & $\boldsymbol{x}$ & $\boldsymbol{x}$ & $\checkmark$ \\
\hline Dynamic and Smart consents & $\checkmark$ & $\boldsymbol{x}$ & $\boldsymbol{x}$ & $\boldsymbol{x}$ & $\boldsymbol{x}$ & $\checkmark$ \\
\hline
\end{tabular}

Note that data analytics is an integral part of the precision health system for various purposes, including prediction, diagnosis, and precise treatment of diseases. Now firstly, a researcher/analyst prepares a model based on the available information at his/her ends. Afterward, he/she submits the model to the coordinating server. Secondly, the coordinating server checks the validity of the modeler, including ethics approval at his/her end. Then, the server and data holders execute the policies, dynamic consent, and smart contracts separately, and update their policies as needed. If the executions return true values, then the coordinating server transmits the received model, which is considered as a global model, to all participating data holders/sources (a data holder can be an institution's private server or cloud or a device such as a mobile phone). Thirdly, after receiving the global model, each data holder trains the model on their local datasets and sends the trained model or updates to the coordinating server in an encrypted form. Fourthly, after receiving all the encrypted private updates from the participating data holders, the coordinating server securely aggregates the updates in its secure enclave provided by Intel SGX, and it updates its previous global model. Afterward, a differential privacy measure, called global differential privacy, is applied to the updated global model's parameters and send back to the clients. This privacy measure ensures the guaranteed privacy of the local data at some cost of performance from the attacks, including membership inference [239|240]241. This process of federated training takes place continuously until the convergence of model parameters. Finally, a trained global model or result is sent back to the researcher/analyst, who uses this model to analyze the health condition of its data subjects. Besides, the individual participants can utilize the final global model for their health analytics. In the above process, the clients can take differential privacy measures to their individual updates to the coordinating server. This is known as local differential privacy measures. However, the coordinating server's operations are confined within the trusted platform intelSGX (i.e., a trusted platform), the local differential privacy is not required. Due to composition property, it reduces the overall accuracy than by using global differential privacy because the server computes on the noisy (differentially private) local updates. 
There are various challenges in the implementation of the presented system. Some of the difficulties are due to the participants with different computing power, low computing speed, dropped connections, data heterogeneity, high communication cost, and malicious participation. Firstly, splitting the model for its distributed training, such as in SplitNN 209 or a variant of SplitNN 238, is a possible solution for participants with low computing power. This is because each participant is responsible for training only a part of the model. However, we may require other models besides neural networks in the platform. Secondly, regarding low computing participants, the addition of trusted workers (like in the case of large batch synchronous SGD), who compute and process on behalf of the participant, in the network can be a solution. This way, we can reduce the reporting time of stragglers to the coordinating server. The statistical challenges, including unbalanced data (non-IID data and variation in data points with time) on each participant, and systems challenges, including high communication cost and issues such as stragglers, are addressed through federated multi-task learning framework, e.g., MOCHA [242]. Besides, asynchronous updating methods address the problems due to stragglers in the network. Thirdly, one can select a sufficient number of participants to avoid the dropped connections. Lastly, how to prevent malicious participation effectively in the federated setting is still an open problem. However, a strengthened auditing system (which performs various activities including accuracy auditing and log checking) and adversarial training 243] enable the anomaly detection during model training and secure aggregation.

\section{Conclusion}

Precision health $(\mathrm{PH})$ data security, privacy, and trust are of utmost importance due to ethical and regulatory requirements, and they are equally demanded by the data owners/subjects. A detailed requirement for the data security and privacy enable to studying and implementing compliance-friendly techniques whilst handling PH data. In this regard, this paper investigated both the regulations (e.g., HIPAA USA, GDPR EU, and Privacy Act Australia) and ethical guidelines to present a detailed requirement for $\mathrm{PH}$ data security and privacy. Our studies observed proper informed consent, secure data transfer, secure and privacy-preserving data processing (computation), proper administrative, physical and technical safeguards, confidentiality, integrity checks, transparency, fairness, availability, minimum and limited use, and breach notification as major requirements from regulatory perspectives. From ethical aspects, studies observed ethics approval, awareness, control, maintaining ownership, limiting information leakage, proper data-sharing management, risk analysis and management along with requirements from regulations, including privacy, security, confidentiality, trust, and breach notification, as essential requirements. Besides, considering the sensitivity of PH data usage in health decision making, health data trustworthiness is a particular requirement. This requires $\mathrm{PH}$ data in a standard format, simple, clear, complete, accurate, timely, and transparent for its effectiveness and correctness whilst making inferences for decisions. We analyzed the challenges based on these requirements, and identified four significant challenges; they are health data security and privacy whilst computing, consent management, PH data trustworthiness, and legal and ethical compliance.

Data analytic is an integral part of precision health. This field is evolving with privacy-preserving techniques that are recently introduced and not discussed jointly in the literature. So, we focused our studies on the relevant techniques and recent developments, including health projects, to address PH data security and privacy whilst computing. Our survey found that there are no significant difficulties for the security and privacy of PH data-at-rest and data-in-transit; however, privacy and security of PH data-in-use are still an active research field. In this aspect, we presented and discussed hardware-based techniques, i.e., trusted execution environment, cryptography-based techniques such as homomorphic encryption and multi-party computation, and differential privacy. By providing an overview of machine learning paradigms in healthcare, our studies suggested No-peek learning as a suitable datacentric and privacy-preserving ML computing technique for $\mathrm{PH}$ data. This is due to its model-to-data computing approach on distributed $\mathrm{PH}$ data (remained in silos).

To provide an overview and practicality of the techniques that we discussed, we reviewed some notable health projects and their techniques for the security and privacy of health data. Our studies observed that blockchain, cryptography, and hardware-based techniques, differential privacy, and policies for consent and privacy are most commonly implemented. Together with the model-to-data approach, we illustrated an example case for the security and privacy of $\mathrm{PH}$ data along with a policy enforcer in a $\mathrm{PH}$ platform. Clearly, these techniques enable the trustworthiness and facilitate ethical clearance, consent management, medical innovations, and developments by handling PH data with compliance. As security threats and maturity of the techniques evolve with time, a study of techniques for security and privacy of PH data-in-use is still an active field of research. 


\section{References}

1. USA UCLA Health. What is precision health? Report, May 2016. https://www.uclahealth.org/precision-health/ what-is-precision-health

2. Standford Medicine, USA. Precision health. Report, 2016. https://stanmed.stanford.edu/content/dam/sm/stanmed/ documents/2016winter.pdf.

3. Sophia Miryam Schssler-Fiorenza Rose, Kvin Contrepois, Kegan J. Moneghetti, Wenyu Zhou, Tejaswini Mishra, Samson Mataraso, Orit Dagan-Rosenfeld, Ariel Ganz, Jessilyn Dunn, Daniel Hornburg, Shannon Rego, Dalia Perelman, Sara Ahadi, Reza Sailani, Yanjiao Zhou, Shana R. Leopold, Jieming Chen, Melanie Ashland, Jeffrey Christle, and Michael P. Snyder. A longitudinal big data approach for precision health. Nature Medicine, 25:792-804, May 2019.

4. Jun Seok Kang, Polina Kuznetsova, Michael Luca, and Yejin Choi. Where not to eat? improving public policy by predicting hygiene inspections using misc reviews. Empirical Methods in Natural Language Processing, 22:58-77, Sept. 2013.

5. OECD/WHO (2018). Health at a glance: Asia/pacific 2018: Measuring progress towards universal health coverage. OECD Publishing, 2018. https://doi.org/10.1787/health_glance_ap_2018_en", last accessed on May 6, 2019.

6. CMS. National health expenditure projections 2018-2027. Report, 2019. https://www.cms.gov/ResearchStatistics-Data-and-Systems/Statistics-Trends-and-Reports/NationalHealthExpendData/Downloads/ ForecastSummary.pdf, last accessed on Dec 6, 2019.

7. Health expenditure Australia 2016-17, Sep. 2018. https://www.aihw.gov.au/reports/health-welfare-expenditure/ health-expenditure-australia-2016-17/contents/summary, last accessed on Jul 03, 2019.

8. The Digital Universe of Opportunities: Rich Data and the Increasing Value of the Internet of Things, Apr. 2014. https://www.emc.com/collateral/analyst-reports/idc-digital-universe-2014.pdf, last accessed on Jun $03,2019$.

9. Allan F. Simpao, Luis M. Ahumada, Jorge A. Galvez, and Mohamed A. Rehman. A review of analytics and clinical informatics in health care. J. Med. Syst. (2014) 38:45, Apr. 2014.

10. N. Coudray, P. S. Ocampo, T. Sakellaropoulos, N. Narula, M. Snuderl, D. Feny, A. L. Moreira, N. Razavian, and A. Tsirigos. Classification and mutation prediction from nonsmall cell lung cancer histopathology images using deep learning. Nature Medicine, 24:1559-1567, Oct. 2018.

11. Marzyeh Ghassemi, Leo Anthony Celi, and David J Stone. State of the art review: the data revolution in critical care. Critical Care (2015) 19:118, Mar. 2015.

12. Clemens Scott Kruse, Rishi Goswamy, Yesha Raval, and Sarah Marawi. Challenges and opportunities of big data in health care: A systematic review. JMIR Med. Inform. 2016;4(4):e38, Nov. 2016.

13. Stanford Medicine. Harnessing the power of data in health. Stanford Medicine 2017 Health Trends Report, Jun. 2017.

14. Diego Ardila, Atilla P. Kiraly, Sujeeth Bharadwaj, Bokyung Choi, Joshua J. Reicher, Lily Peng, Daniel Tse, Mozziyar Etemadi, Wenxing Ye, Greg Corrado, David P. Naidich, and Shravya Shetty. End-to-end lung cancer screening with three-dimensional deep learning on low-dose chest computed tomography. Nature Med., May 2019.

15. Healthcare analytics market to hit \$31 billion by 2022, Aug. 2018.

16. Accenture. Artificial intelligence: Healthcares new nervous system. Accenture Article, Dec. 2017.

17. Patient Engagement Survey 2018, 2018. https://www.medicaldirector.com/resources/patient-engagement-survey2018, last accessed on Jul 03, 2019.

18. OAIC (Aus. Govt.). Notifiable data breaches quarterly statistics report. Quarterly Statistics Report, Feb. 2019. https://www.oaic.gov.au/resources/privacy-law/privacy-act/notifiable-data-breaches-scheme/quarterlystatistics/notifiable-data-breaches-quarterly-statistics-report-1-october-31-december-2018.pdf.

19. HIPAA. 2019 data breach barometer report shows massive increase in exposed healthcare records. HIPAA Journal, Feb. 2019. https://www.hipaajournal.com/2019-data-breach-barometer-report-shows-massive-increasein-exposed-healthcare-records/

20. Stanford Medicine. The democratization of health care. Stanford Medicine 2018 Health Trends Report, Dec. 2018.

21. Forgerock. Us consumer data breach report 2019: Personally identifiable information targeted in breaches that impact billions of records. Forgerock Breach report 2019, Jun. 2019.

22. HIPAA. What is protected health information? HIPAA Journal, Jan. 2018. https://www.hipaajournal.com/what-isprotected-health-information/

23. OAIC (Aus. Govt.). What is personal information? Report, May 2017. https://www.oaic.gov.au/agencies-andorganisations/guides/what-is-personal-information.

24. EU. Regulation (eu) 2016/679 general data protection regulation. Official Journal of the European Union, May 2016.

25. Jos Luis Fernndez-Alemn, Inmaculada Carrin Seor, Pedro ngel Oliver Lozoya, and Ambrosio Toval. Security and privacy in electronic health records: A systematic literature review. Journal of Biomedical Informatics, 46:3:541-562, 2013.

26. Assad Abbas and Samee U. Khan. A review on the state-of-the-art privacy-preserving approaches in the e-health clouds. IEEE JOURNAL OF BIOMEDICAL AND HEALTH INFORMATICS, 18:4, 2014.

27. Karim ABOUELMEHDI, Abderrahim BENI-HSSANE, Hayat KHALOUFI, and Mostafa SAADI. Big data security and privacy in healthcare: A review. EUSPN, pages 73-80, 2017.

28. April Moreno Arellano, Wenrui Dai, Shuang Wang, Xiaoqian Jiang, and Lucila Ohno-Machado. Privacy policy and technology in biomedical data science. Annu Rev Biomed Data Sci., 1:115129., 2018. 
29. Karim Abouelmehdi, Abderrahim BeniHessane, and Hayat Khalouf. Big healthcare data: preserving security and privacy. Journal of big data, 5:1:1-18, 2018.

30. Yazan Al-Issa, Mohammad Ashraf Ottom, and Ahmed Tamrawi. ehealth cloud security challenges: A survey. J Healthc Eng., 2019: 7516035, 2019.

31. JIE XU and FEI WANG. Federated learning for healthcare informatics. arxiv.org, 2019.

32. Md Momin Al Aziz, Md Nazmus Sadat, Dima Alhadidi, Shuang Wang, Xiaoqian Jiang, Cheryl L Brown, and Noman Mohammed. Privacy-preserving techniques of genomic data-a survey. Briefings in Bioinformatics, 20(3):887-895, 2019.

33. Adnan Qayyum, Junaid Qadir, Muhammad Bilal, and Ala Al-Fuqaha. Secure and robust machine learning for healthcare: A survey. arxiv.org, 2020.

34. Georgios A. Kaissis, Marcus R. Makowski, Daniel Rckert, and Rickmer F. Braren. Secure, privacy-preserving and federated machine learning in medical imaging. Nature Machine Intelligence, 2:305311, 2020.

35. Fadila Zerka, Samir Barakat, , Sean Walsh, Marta Bogowicz, Ralph T. H. Leijenaar, Arthur Jochems, Benjamin Miraglio, David Townend, and Philippe Lambin. Systematic review of privacy-preserving distributed machine learning from federated databases in health care. JCO clinical cancer informatics, 4:184 - 200, 2020.

36. Oxford dictionary, 2019. https://en.oxforddictionaries.com/definition/law, last accessed on May $15,2019$.

37. Oxford dictionary, 2019. https://en.oxforddictionaries.com/definition/ethics last accessed on May 15, 2019.

38. C. M. OKeefe and C. J. Connolly. Privacy and the use of health data for research. Medical Journal of Australia, 193(9), Nov. 2010.

39. California Department of Justice. California consumer privacy act (ccpa), 2018. https://oag.ca.gov/privacy/ccpa last accessed on July 7, 2020.

40. HHS. Summary of the hipaa privacy rule. HIPAA Compliance Assistance, Jul. 2013.

41. HHS. Summary of the hipaa security rule. HIPAA Compliance Assistance, Jul. 2013.

42. GINA. H.r. 493 (110th): Genetic information nondiscrimination act of 2008. Report, May 2008. https:// www.govtrack.us/congress/bills/110/hr493/text, last accessed on Jun 27, 2019.

43. HITECH. Health information technology for economic and clinical health act. Report, Feb. 2009. https://www.hhs.gov/ sites/default/files/ocr/privacy/hipaa/understanding/coveredentities/hitechact.pdf last accessed on Jun 27, 2019.

44. EU. Declaration on ethics and data protection in artifical intelligence. In Int. Conf. of Data Protection 63 Privacy Commissioners, ICDPPC, pages 1-6, Oct. 2018.

45. The Australian Government. Privacy act 1988. Federal Register of Legislation, Compilation No. 80, Jan 2019.

46. The Australian Government. Australian privacy principles guidelines: Privacy act 1988. APP Guidelines, Mar. 2015.

47. The Australian Government. My health records act 2012. Federal Register of Legislation, Compilation No. 7, Sept. 2017.

48. World Health Organization. Legal frameworks for ehealth. Report, 5, 2012. https://apps.who.int/iris/bitstream/ handle/10665/44807/9789241503143_eng.pdf; jsessionid=CF0A764CC36397B44FCCB4DC7F415238?sequence=1, last accessed on July $7,2020$.

49. European Economic and Social Committee. The ethics of big data: Balancing economic benefits and ethical questions of big data in the eu policy context. Report, 2017. https://www.eesc.europa.eu/sites/default/files/resources/ docs/qe-04-17-306-en-n.pdf, last accessed on July 7, 2020.

50. NHMRC, ARC, and UA. The national statement on ethical conduct in human research 2007 (updated 2018). Report, 2007. https://www.nhmrc.gov.au/about-us/publications/national-statement-ethical-conduct-human-research2007-updated-2018\#block-views-block-file-attachments-content-block-1, last accessed on July 3, 2020.

51. AMA. Augmented intelligence in health care. Report, 2019. https://www.ama-assn.org/system/files/2019-01/ augmented-intelligence-policy-report.pdf

52. WMA. Wma declaration of helsinki ethical principles for medical research involving human subjects, 2018. https://www.wma.net/policies-post/wma-declaration-of-helsinki-ethical-principles-for-medicalresearch-involving-human-subjects/, last accessed on July 7, 2020.

53. Farag Azzedin and Mustafa Ghaleb. Internet-of-things and information fusion: Trust perspective survey. Sensors (Basel, Switzerland), 19(8):1929, 2019.

54. Dimensional Insight. Understanding data trust in healthcare: A cios perspective, $2018 . \quad$ https:// wWw.anesthesiallc.com/images/eAlertsSource/data_trust_survey_2018_110718-min.pdf, last accessed on July 8, 2020.

55. WHO. Improving data quality: A guide for developing countries. Report, $2003 . \quad$ http:// www.globalhealthworkforce.org/resources/who_improving_data_quality.pdf.

56. Sion Davoudi, Julie A Dooling, and Barbara Glondys. Data quality management model (2015 update) - retired. Journal of AHIMA, 86, no. 10, 2015.

57. Mark D Wilkinson, Michel Dumontier, and I. Jan Aalbersberg et al. The fair guiding principles for scientific data management and stewardship. Sci. Data, 3, 160018, 2016.

58. Joan Daemen and Vincent Rijmen. Advanced encryption standard (aes). Federal Information Processing Standards Publications (FIPS PUBS) 19\%, Nov. 2001.

59. R. L. Rivest, A. Shamir, and L. Adleman. A method for obtaining digital signatures and public-key cryptosystems. Communications of the ACM, 21(2):120-126, 1978. 
60. N. Koblitz. Elliptic curve cryptosystems. Mathematics of Computation, 48(177), Jan. 1987.

61. CHRISTOPH BOSCH, PIETER HARTEL, WILLEM JONKER, and ANDREAS PETER. A survey of provably secure searchable encryption. ACM Computing Surveys, 47(2):18:18-51, 2014.

62. Internet Engineering Task Force (IETF). The transport layer security (TLS) protocol version 1.3. IETF proposed standard, Aug. 2018.

63. Network Working Group. Securing FTP with TLS. Proposed standard, Oct. 2005. https://tools.ietf.org/html/ rfc4217

64. Abigail Goldsteen, Shelly Garion, Sima Nadler, Natalia Razinkov, Yosef Moatti, and Paula Ta-Shma. Brief announcement: A consent management solution for enterprises. Cyber Security Cryptography and Machine Learning CSCML 2017, 10332:189-192, Jun 2017.

65. Isabelle Budin-Ljsne and Harriet J. A. et al. Teare. Dynamic consent: a potential solution to some of the challenges of modern biomedical research. BMC Medical Ethics, 18, 4, 2017.

66. Jane Kaye, Edgar A Whitley, David Lund, Michael Morrison, Harriet Teare, and Karen Melham. Dynamic consent: a patient interface for twenty-first century research networks. European Journal of Human Genetics, 23(2):141-146, 2015.

67. Tatiana Ermakova, Benjamin Fabian, and Rdiger Zarnekow. Acceptance of health clouds - a privacy calculus perspective. In Proc. of the European Conf. on Information Systems (ECIS) 2014, pages 1-13, 2014.

68. OAIC (Aus. Govt.). Guide to data analytics and the australian privacy principles. Report, Mar. 2018. https://www.oaic.gov.au/resources/agencies-and-organisations/guides/guide-to-data-analytics-andthe-australian-privacy-principles.pdf

69. Aus. Govt. OAIC. Guide to undertaking privacy impact assessments. Report, 2014. https://www.oaic.gov.au/ resources/agencies-and-organisations/guides/guide-to-undertaking-privacy-impact-assessments.pdf

70. Aus. Govt. NHMRC. Guidelines approved under section 95a of the privacy act 1988 . Report, 2014. https: //www.nhmrc.gov.au/about-us/publications/guidelines-approved-under-section-95a-privacy-act-1988.

71. A. J. Menezes, P. C. van Oorschot, and S. A. Vanstone. Handbook of Applied Cryptography. CRC Press, 5th ed. edition, 2001.

72. Gemalto. Data privacy and new regulations take center stage. 2018 First Half Review, 2018.

73. Microsoft. Overview of bitlocker device encryption in windows 10. Report, Jan. 2019. https://docs.microsoft.com/enus/windows/security/information-protection/bitlocker/bitlocker-device-encryption-overview-windows-10.

74. IDRIX, 2020. https://www.veracrypt.fr/en/Introduction.html, last accessed on May 5, 2020.

75. SEAGATE, 2020. https://www.seagate.com/au/en/tech-insights/protect-data-with-seagate-secure-selfencrypting-drives-master-ti/.

76. J. A. Halderman, S. D. Schoen, N. Heninger, W. Clarkson, W. Paul, J. A. Calandrino, A. J. Feldman, J. Appelbaum, and E. W. Felten. Lest we remember: cold boot attacks on encryption keys. In Proc. USENIX Security Symp., pages 45-60, Jul. 2008.

77. NIST. Guide to storage encryption technologies for end user devices. NIST Special Publication 800-111, Nov. 2007.

78. Michael Henson and Stephen Taylor. Memory encryption: A survey of existing techniques. ACM Comput. Surv., 46, Mar. 2014.

79. Lilian Bossuet, Michael Grand, Lubos Gaspar, Viktor Fischer, and Guy Gogniat. Architectures of flexible symmetric key crypto enginesa survey: From hardware coprocessor to multi-crypto-processor system on chip. ACM Comput. Surv., 45(41), Aug. 2013.

80. Guardtime Federal, LLC. Keyless signature infrastructure (ksi) technology: An introduction to ksi blockchain technology and its benefits. White paper, 2017. http://blockchain.machetemag.com/wp-content/uploads/2017/11/ Guardtime_WhitePaper_KSI.pdf

81. e-estonia, 2019. https://e-estonia.com/solutions/healthcare/, last accessed on Jul 04, 2019.

82. Jun. 2018. https://guardtime.com/blog/world-s-first-blockchain-supported-personal-care-recordplatform-launched-by-guardtime-and-partners, last accessed on Jul 04, 2019.

83. William J. Gordon and Christian Catalini. Blockchain technology for healthcare: facilitating the transition to patientdriven interoperability. Comput. Struct. Biotechnol. J., 16:224230, 2018.

84. Dennis Grishin, Jean Louis Raisaro, and Juan Ramn Troncoso-Pastoriza et al. Citizen-centered, auditable, and privacypreserving population genomics. bioRxiv, 2019. https://www.biorxiv.org/content/10.1101/799999v1

85. Cornelius C. Agbo, Qusay H. Mahmoud, and J. Mikael Eklund. Blockchain technology in healthcare: A systematic review. Healthcare(Basel), 7(2):56:1-30, 2019.

86. Verizon. 2018 data breach investigations report. Research report, 2018. http://www.documentwereld.nl/files/2018/ Verizon-DBIR_2018-Main_report.pdf.

87. F. Sabahi and A. Movaghar. Intrusion detection: A survey. In 2008 Third Int. Conf. on Systems and Networks Commun., pages 23-26, Oct 2008 .

88. D. Stiawan, A. H. Abdullah, and M. Yazid Idris. The trends of intrusion prevention system network. In $20102 n d$ Int. Conf. on Education Tech. and Computer, volume 4, pages 217-221, June 2010.

89. Joseph Migga Kizza. Guide to Computer Network Security. Springer, Cham, 4th ed. edition, 2017.

90. EU. Opinion 05/2014 on anonymisation techniques. ARTICLE 29 DATA PROTECTION WORKING PARTY, 2014.

91. Luc Rocher, Julien M. Hendrickx, and Yves-Alexandre de Montjoye. Estimating the success of re-identifications in incomplete datasets using generative models. Nature Communications, 10(1):1 -9, Jul. 2019. 
92. Sara Porat, Boaz Carmeli, Tamar Domany, Tal Drory, Ksenya Kveler, Alex Melament, and Haim Nelken. Masking gateway for enterprises. Languages: From Formal to Natural, 5533:177-191, 2009.

93. ARM. ARM security technology - building a secure system using trustzone technology. ARM white Paper, 2009.

94. Victor Costan and Srinivas Devadas. Intel SGX explained. Cryptology ePrint Archive, Report 2016/086, 2016.

95. TCG. TPM main part 1 design principles. TCG Specification Version 1.2 Revision 116, Mar. 2011.

96. Intel, 2019. https://www.intel.com.au/content/www/au/en/support/articles/000025873/technologies.html last accessed on May 26, 2019.

97. AMD. AMD memory encryption. AMD White Paper, Apr. 2016.

98. Victor Costan, Ilia Lebedev, and Srinivas Devadas. Sanctum: Minimal hardware extensions for strong software isolation. In 25th USENIX Security Symposium (USENIX Security 16), pages 857-874. USENIX Association, 2016.

99. Keystone project group, 2018. http://docs.keystone-enclave.org/en/latest/Getting-Started/index.html, last accessed on May 9, 2019.

100. SANDRO PINTO and NUNO SANTOS. Demystifying arm trustzone: A comprehensive survey. ACM Comput. Surv., 51(6):130:1-130:36, Jan. 2019.

101. Intel. Intel platform developers kit for sgx, 2020. https://designintools.intel.com/ Intel_Platform_Developers_Kit_for_SGX_p/q6uidcsgxpdk.htm, last accessed on Jan 29, 2020.

102. Fortanix. Fortanix enclave development platform, 2019. https://github.com/fortanix/rust-sgx last accessed on Jan 29,2020 .

103. Microsoft Openenclave team. Open enclave sdk, 2019. https://github.com/openenclave/openenclave, last accessed on Jan 29, 2020.

104. Intel. Intel software guard extensions ssl, 2019. https://github.com/intel/intel-sgx-ssl\#intel-software-guardextensions-ssl, last accessed on Jan 29, 2020.

105. Wenhao Wang, Guoxing Chen, Xiaorui Pan, Yinqian Zhang, XiaoFeng Wang, Vincent Bindschaedler, Haixu Tang, and C. A. Gunter. Leaky cauldron on the dark land: Understanding memory side-channel hazards in sgx. In Proc. of the 2017 ACM SIGSAC Conf. on Comp. and Commun. Security, pages 2421-2434, Oct. 2017.

106. Mariacarla Staffa, Luigi Coppolino, Luigi Sgaglione, Erol Gelenbe, Ioannis Komnios, Evangelos Grivas, Oana Stan, and Luigi Castaldo. KONFIDO: An openNCP-based secure ehealth data exchange system. In Gelenbe E. et al. (eds) Security in Computer and Information Sciences, Euro-CYBERSEC 2018, Communications in Computer and Information Science, volume 821, pages 11-27, Jul. 2018.

107. Feng Chen, Shuang Wang, Xiaoqian Jiang, Sijie Ding, Yao Lu, Jihoon Kim, S Cenk Sahinalp, Chisato Shimizu, Jane C Burns, Victoria J Wright Eileen Png, Martin L Hibberd, David D Lloyd, Hai Yang, Amalio Telenti, Cinnamon S Bloss, Dov Fox, Kristin Lauter, and Lucila Ohno-Machado. PRINCESS: Privacy-protecting rare disease international network collaboration via encryption through software guard extensions. Bioinformatics, 33(6):871-878, Mar. 2017.

108. ARM. Security for the next one trillion devices, 2019. https://www.arm.com/why-arm/security, last accessed on Jan 30, 2020.

109. Abbas Acar, Hidayet Aksu, A. S. Uluagac, and Mauro Conti. A survey on homomorphic encryption schemes: Theory and implementation. ACM Comput. Surv., 51(4):79:1-79:35, Jul. 2018.

110. Shafi Goldwasser and Silvio Micali. Probabilistic encryption \& how to play mental poker keeping secret all partial information. In 14th Annual ACM Symp. on Theory of Comput., pages 365-377, 1982.

111. Akinori Kawachi, Keisuke Tanaka, and Keita Xagawa. Multi-bit cryptosystems based on lattice problems. In Public Key Cryptography, pages 315-329, 2007.

112. Andrew Chi-Chih Yao. Protocols for secure computations. In FOCS, volume 82, pages 160-164, 1982.

113. T. Sander, A. Young, and M. Yung. Non-interactive cryptocomputing for nc1. In 40th Annual Symp. on Found. of Comp. Science, pages 554-566, Oct. 1999.

114. Yuval Ishai and Anat Paskin. Evaluating branching programs on encrypted data. In Proc. of the 4 th conference on Theory of cryptography, pages 575-594, Feb. 2007.

115. Craig Gentry. Fully homomorphic encryption scheme. Doctoral Dissertation, 2009.

116. Ilaria Chillotti, Nicolas Gama, Mariya Georgieva, and Malika Izabachene. Faster fully homomorphic encryption: Bootstrapping in less than 0.1 seconds. In Advances in Cryptology - ASIACRYPT 2016, pages 3-33, Nov. 2016.

117. Shai Halevi and Victor Shoup. Faster homomorphic linear transformations in helib. Advances in Cryptology - CRYPTO 2018, Lecture Notes in Computer Science, 10991:93-120, Jul. 2018.

118. Florian Kerschbaum. Privacy-preserving computation. In Revised Selected Papers of the First Annual Privacy Forum on Privacy Technologies and Policy - Volume 8319, APF 2012, pages 41-54, 2014.

119. Zvika Brakerski, Craig Gentry, and Vinod Vaikuntanathan. (leveled) fully homomorphic encryption without bootstrapping. In Proc. of the 3rd Innovations in Theoretical Comp. Science Conf., ITCS '12, pages 309-325, 2012.

120. Jean Louis Raisaro, Gwangbae Choi, Sylvain Pradervand, Raphael Colsenet, Nathalie Jacquemont, Nicolas Rosat, Vincent Mooser, and Jean-Pierre Hubaux. Protecting privacy and security of genomic data in i2b2 with homomorphic encryption and differential privacy. IEEE/ACM TRANSACTIONS ON COMPUTATIONAL BIOLOGY AND BIOINFORMATICS, 15(5):1413-1426, Jul. 2018.

121. Joppe W. Bos, Kristin Lauter, and Michael Naehrig. Private predictive analysis on encrypted medical data. Journal of Biomed. Inform., pages 234-243, May 2014.

122. Jun Jie Sim, Fook Mun Chan, Shibin Chen, Benjamin Hong Meng Tan, and Khin Mi Mi Aung. Achieving gwas with homomorphic encryption. BMC Med Genomics, 13(Suppl 7):90:1-12, 2020. 
123. A. C. Yao. Protocols for secure computations. In Proc. 23th Annual Symp. on Found. of Comput. Science (FOCS 82), pages 160-164, Nov. 1982.

124. M. Ben-Or, S. Goldwasser, and A. Wigderson. Completeness theorems for noncryptographic fault-tolerant distributed computations. In Proc. 20th Annual ACM Symp. on Theory of Comput., STOC '88, pages 1-10, 1988.

125. I. Damgard, V. Pastro, N. Smart, and S. Zakarias. Multiparty computation from somewhat homomorphic encryption. In Advances in Cryptology CRYPTO 2012, LNCS 7417, pages 643-662, 2012.

126. Adi Shamir. How to share a secret. Commun. ACM, 22(11):612-613, Nov. 1979.

127. Craig Gentry, K. A. Goldman, Shai Halevi, Charanjit Julta, Mariana Raykova, and Daniel Wichs. Optimizing oram and using it efficiently for secure computation. In Proc. Inter. Symp. on Privacy Enhancing Tech. Symp., PETS 2013: Privacy Enhancing Technologies, pages 1-18, 2013.

128. S. Zahur, X. Wang, M. Raykova, A. Gascon, J. Doerner, D. Evans, and J. Katz. Revisiting square-root oram: Efficient random access in multi-party computation. In 2016 IEEE Symp. on Security and Privacy (SP), pages 218-234, May 2016.

129. Sameer Wagh, Divya Gupta, and Nishanth Chandran. Securenn: Efficient and private neural network training. IACR Cryptology ePrint Archive, 2018:442, 2018.

130. David Chaum, Ivan B. Damgard, and Jeroen van de Graaf. Multiparty computations ensuring privacy of each party's input and correctness of the result. In A Conf. on the Theory and Applications of Cryptographic Tech. on Advances in Cryptolog, CRYPTO'87, pages 87-119, 1988.

131. SODA. Scalable oblivious data analytics, 2019. https://www.soda-project.eu/, last accessed on Jul $2,2019$.

132. NTT. Trial service of seucure computation system san-shi. NTT Press Releases, 2018.

133. NTT. The world's first demonstration of secret computing technology in medical statistical processing. NTT Press Releases, Feb. 2012.

134. Peeter Laud and Alisa Pankova. Privacy-preserving record linkage in large databases using secure multiparty computation. BMC Medical Genomics, 11(4), Oct. 2018.

135. Dan Bogdanov, Sven Laur, and Jan Willemson. Sharemind: A framework for fast privacy-preserving computations. In Proc. of the 13th European Symp. on Research in Comput. Security: Computer Security, ESORICS '08, pages 192-206, 2008.

136. Cynthia Dwork. Differential privacy. In Proc. of the 33rd Int. Conf. on Automata, Languages and Programming Volume Part II, ICALP'06, pages 1-12, 2006.

137. Cynthia Dwork, Frank McSherry, Kobbi Nissim, and Adam Smith. Calibrating noise to sensitivity in private data analysis. In Proc. of the Third Conf. on Theory of Cryptography, TCC'06, pages 265-284, 2006.

138. F. McSherry and K. Talwar. Mechanism design via differential privacy. In 48th Annual IEEE Symp. on Found. of Comp. Science (FOCS'O7), pages 94-103, Oct 2007.

139. Cynthia Dwork and Aaron Roth. The algorithmic foundations of differential privacy. Foundations and Trends in Theoretical Computer Science, 9(34):211-407, 2014.

140. Chris Clifton and Tamir Tassa. On syntactic anonymity and differential privacy. In 2013 IEEE 29th Int. Conf. on Data Eng. Workshops (ICDEW), pages 88-93, April 2013.

141. J. L. Raisaro, J. Troncoso-Pastoriza, M. Misbach, J. Sa Sousa, S. Pradervand, E. Missiaglia, O. Michielin, B. Ford, and J. Hubaux. Medco: Enabling secure and privacy-preserving exploration of distributed clinical and genomic data. IEEE/ACM Transactions on Computational Biology and Bioinformatics, 2018.

142. D. Vu and A. Slavkovic. Differential privacy for clinical trial data: Preliminary evaluations. In 2009 IEEE Int. Conf. on Data Mining Workshops, pages 138-143, Dec. 2009.

143. Brett K. Beaulieu-Jones, William Yuan, Samuel G. Finlayson, and Zhiwei Steven Wu. Privacy-preserving distributed deep learning for clinical data. In Machine Learning for Health Workshop at NeurIPS 2018, pages 1-6, Dec. 2018.

144. Ilya Mironov. Rnyi differential privacy. In 30th IEEE Comp. Security Found. Symp., CSF 2017, pages 263-275, Aug. 2017.

145. Genomics england, 2012. https://www.genomicsengland.co.uk/about-genomics-england/the-100000-genomesproject/, last accessed on May 28, 2019.

146. 23andMe, 2019. https://www.23andme.com/en-int/dna-ancestry/ last accessed on May 15, 2019.

147. Global Alliance for Genomics \& Health, 2013. https://www.ga4gh.org/about-us/, last accessed on May $15,2019$.

148. Personalized Health Informatics Group, 2017. https://www.sphn.ch/en/about.html, last accessed on May $15,2019$.

149. MedCo team, 2019. https://medco.epfl.ch/, last accessed on May 15, 2019.

150. Justin Guinney and Julio Saez-Rodriguez. Alternative models for sharing confidential biomedical data. Nature Bio Tech., 36(5):391-392, May 2018.

151. Australian Institute of Health and Welfare, 2019. https://www.aihw.gov.au/about-our-data/accessing-australiangovernment-data, last accessed on May 28, 2019.

152. HealthData.gov, 2019. https://healthdata.gov/search/field_topic/health-17816/type/dataset?sort_by= changed, last accessed on May 28, 2019.

153. European Data Portal, 2019. https://www.europeandataportal.eu/data/\#/datasets?categories=heal\&page= $1 \&$ locale $=$ en, last accessed on May 28, 2019.

154. M. J. Sheller, G. A. Reina, Brandon Edwards, Jason Martin, and Spyridon Bakas. Multi-institutional deep learning modeling without sharing patient data: A feasibility study on brain tumor segmentation. CoRR, abs/1810.04304, 2018. 
155. Li Huang and Dianbo Liu. Patient clustering improves efficiency of federated machine learning to predict mortality and hospital stay time using distributed electronic medical records. CoRR, abs/1903.09296, 2019.

156. The Apache software foundation, 2019. http://hadoop.apache.org/, last accessed on May 30, 2019.

157. Amazon, 2019. https://aws.amazon.com/ec2/, last accessed on May 30, 2019.

158. Satoshi Nakamoto. Bitcoin: A peer-to-peer electronic cash system. White paper, 2008. https://bitcoin.org/ bitcoin.pdf

159. iExec. Blockchain-based decentralized cloud computing. White paper, Apr. 2018. https://iex.ec/wp-content/ uploads/pdf/iExec-WPv3.0-English.pdf.

160. golem team, 2019. https://docs.golem.network/\#/About/What-is-Golem, last accessed on May 30, 2019.

161. Sinno Jialin Pan and Qiang Yang. A survey on transfer learning. IEEE Trans. on Knowl. and Data Eng., 22(10):13451359, Oct. 2010.

162. Yann LeCun, Yoshua Bengio, and Geoffrey Hinton. Deep learning. Nature, 521:436-444, May. 2015.

163. Michael McCloskey and Neal J. Cohen. Catastrophic interference in connectionist networks: The sequential learning problem. The Psychology of Learning and Motivation, 24:109-165, Jul. 1989.

164. Zhizhong Li and Derek W Hoiem. Learning without forgetting. In Nicu Sebe, Bastian Leibe, Max Welling, and Jiri Matas, editors, Proc. Computer Vision - 14th European Conference ECCV 2016, Lecture Notes in Computer Science (including subseries Lecture Notes in Artificial Intelligence and Lecture Notes in Bioinformatics), pages 614629. Springer-Verlag, Jan. 2016.

165. Andrei A. Rusu, Neil C. Rabinowitz, Guillaume Desjardins, Hubert Soyer, James Kirkpatrick, Koray Kavukcuoglu, Razvan Pascanu, and Raia Hadsell. Progressive neural networks. CoRR, abs/1606.04671, Aug. 2016. http://arxiv.org/ abs/1606.04671

166. James Kirkpatrick, Razvan Pascanu, Neil Rabinowitz, Joel Veness, Guillaume Desjardins, Andrei A. Rusu, Kieran Milan, John Quan, Tiago Ramalho, Agnieszka Grabska-Barwinska, Demis Hassabis, Claudia Clopath, Dharshan Kumaran, and Raia Hadsell. Overcoming catastrophic forgetting in neural networks. Proc. of the National Academy of Sciences, 114(13):3521-3526, 2017.

167. Chuen-Kai Shie, Chung-Hisang Chuang, Chun-Nan Chou, Meng-Hsi Wu, and Edward Y. Chang. Transfer representation learning for medical image analysis. In Proc. Annual Int. Conf. of the IEEE Eng. in Med. and Biology Society, pages 711-714, Aug. 2015.

168. Hoo chang Shin, Holger Roth, Mingchen Gao, Le Lu, Ziyue Xu, Isabella Nogues, Jianhua Yao, Daniel Mollura, and Ronald Summers. Deep convolutional neural networks for computer-aided detection: Cnn architectures, dataset characteristics and transfer learning. IEEE Transactions on Medical Imaging, 35, Feb. 2016.

169. J. Deng, W. Dong, R. Socher, L. Li, Kai Li, and Li Fei-Fei. Imagenet: A large-scale hierarchical image database. In 2009 IEEE Conf. on Comp. Vision and Pattern Recognition, pages 248-255, Jun. 2009.

170. C. Szegedy, Wei Liu, Yangqing Jia, P. Sermanet, S. Reed, D. Anguelov, D. Erhan, V. Vanhoucke, and A. Rabinovich. Going deeper with convolutions. In 2015 IEEE Conf. on Comp. Vision and Pattern Recog. (CVPR), pages 1-9, June 2015.

171. Alex Krizhevsky, Ilya Sutskever, and Geoffrey E. Hinton. Imagenet classification with deep convolutional neural networks. Commun. ACM, 60(6):84-90, May 2017.

172. Mark Christopher, Akram Belghith, Christopher Bowd, James A. Proudfoot, Michael H. Goldbaum, Robert N. Weinreb, Christopher A. Girkin, Jefrey M. Liebmann, and Linda M. Zangwill. Performance of deep learning architectures and transfer learning for detecting glaucomatous optic neuropathy in fundus photographs. Nature Scientific Reports, 8(16685), Nov. 2018.

173. Rich Caruana. Multitask learning. Mach. Learn., 28(1):41-75, Jul. 1997.

174. Yu Zhang and Qiang Yang. A survey on multi-task learning. CoRR, abs/1707.08114, Jul. 2018. http://arxiv.org/ abs/1707.08114

175. Bharath Ramsundar, Steven Kearnes, Patrick Riley, Dale Webster, David Konerding, and Vijay Pande. Massively multitask networks for drug discovery. CoRR, abs/1502.02072, Feb. 2015. https://arxiv.org/pdf/1502.02072.pdf

176. Jiayu Zhou, Lei Yuan, Jun Liu, and Jieping Ye. A multi-task learning formulation for predicting disease progression. In Proc. of the 17th ACM SIGKDD Int. Conf. on Knowledge Discovery and Data Mining, KDD '11, pages 814-822, 2011.

177. Adrian Benton, Margaret Mitchell, and Dirk Hovy. Multitask learning for mental health conditions with limited social media data. In Proc. of the 15th Conf. of the European Chapter of the Assoc. for Computational Linguistics, volume 1, pages 152-162, Apr. 2017.

178. Rich Caruana, Shumeet Baluja, and Tom Mitchell. Using the future to "Sort out" the present: Rankprop and multitask learning for medical risk evaluation. In Proc. of the 8th Int. Conf. on Neural Info. Processing Systems, NIPS'95, pages 959-965, 1995.

179. Hrayr Harutyunyan, Hrant Khachatrian, David C. Kale, Greg Ver Steeg, and Aram Galstyan. Multitask learning and benchmarking with clinical time series data. Scientific Data, 6(96):1-18, Jun. 2019.

180. J. Ji, X. Chen, C. Luo, and P. Li. A deep multi-task learning approach for ecg data analysis. In 2018 IEEE EMBS Int. Conf. on Biomed. Health Informatics (BHI), pages 124-127, Mar. 2018.

181. Alexander Gepperth and Barbara Hammer. Incremental learning algorithms and applications. In Proc. EU Symp. on Artificial Neural Networks (ESANN), Comput. Intel. and Machine Learning, pages 357-368, Apr. 2016.

182. Haibo He, Sheng Chen, Kang Li, and Xin Xu. Incremental learning from stream data. IEEE Trans. on Neural Net., 22(12):1901-1914, Dec. 2011. 
183. A. LHeureux, K. Grolinger, H. F. Elyamany, and M. A. M. Capretz. Machine learning with big data: Challenges and approaches. IEEE Access, 5:7776-7797, 2017.

184. Daniel Silver, Qiang Yang, and Lianghao Li. Lifelong machine learning systems: Beyond learning algorithms. In $A A A I$ Spring Symposium - Technical Report, pages 49-55, Mar. 2013.

185. A. Misra, A. Sowmya, and P. Compton. Incremental learning for segmentation in medical images. In $3 r d$ IEEE Int. Symp. on Biomed. Imaging: Nano to Macro, 2006., pages 1360-1363, April 2006.

186. Sam Chao and Fai Wong. An incremental decision tree learning methodology regarding attributes in medical data mining. In 2009 Int. Conf. on Machine Learning and Cybernetics, volume 3, pages 1694-1699, Jul. 2009.

187. 2017. https://www.re3data.org/repository/r3d100010960 last accessed on Jun 17, 2019.

188. P. E. Utgoff. An improved algorithm for incremental induction of decision trees. In Proc. of the Eleventh Int. Conf. on Intl. Conf. on Machine Learning, ICML'94, pages 318-325, 1994.

189. Thomas G. Dietterich. Ensemble methods in machine learning. In Proc. of the First Intl. Workshop on Multiple Classifier Systems, MCS '00, pages 1-15, 2000.

190. B. Waske and J. A. Benediktsson. Fusion of support vector machines for classification of multisensor data. IEEE Trans. on Geoscience and Remote Sensing, 45(12):3858-3866, Dec. 2007.

191. Borja Ayerdi, Alexandre Savio, and Manuel Graña. Meta-ensembles of classifiers for alzheimer's disease detection using independent ROI features. In IWINAC (2), volume 7931 of Lecture Notes in Computer Science, pages 122-130. Springer, 2013.

192. Yan Lia, Changxin Baia, and Chandan K. Reddya. A distributed ensemble approach for mining healthcare data under privacy constraints. Information Sciences, 330:245-259, Feb. 2016.

193. Hualong $\mathrm{Yu}$ and Jun Ni. An improved ensemble learning method for classifying high-dimensional and imbalanced biomedicine data. IEEE/ACM Trans. Comput. Biol. Bioinformatics, 11(4):657-666, Jul. 2014.

194. H. B. McMahan, E. Moore, D. Ramage, S. Hampson, and B. A. Arcas. Communication-efficient learning of deep networks from decentralized data. In Proc. of the 20th Int. Conf. on Artificial Intelligence and Statistics (AISTATS), volume 54 of JMLR: WECP, pages 1-10, 2017.

195. Jakub Konecny, H. Brendan McMahan, and Daniel Ramage. Federated optimization: Distributed optimization beyond the datacenter. CoRR, abs/1511.03575, Nov. 2015.

196. Jakub Konecny, H. B. McMahan, Daniel Ramage, and Peter Richtarik. Federated optimization: Distributed machine learning for on-device intelligence. CoRR, abs/1610.02527, Oct. 2016.

197. Keith Bonawitz, Hubert Eichner, Wolfgang Grieskamp, Dzmitry Huba, Alex Ingerman, Vladimir Ivanov, Chloe Kiddon, Jakub Konecny, Stefano Mazzocchi, H. Brendan McMahan, Timon Van Overveldt, David Petrou, Daniel Ramage, and Jason Roselander. Towards federated learning at scale: System design. CoRR, abs/1902.01046, Mar. 2019.

198. Keith Bonawitz, Vladimir Ivanov, Ben Kreuter, Antonio Marcedone, H. Brendan McMahan, Sarvar Patel, Daniel Ramage, Aaron Segal, and Karn Seth. Practical secure aggregation for privacy-preserving machine learning. In Proc. of the 2017 ACM SIGSAC Conf. on Comp. and Commun. Security, CCS '17, pages 1175-1191, 2017.

199. TensorFlow. Tensorflow federated: Machine learning on decentralized data, 2019. https://www.tensorflow.org/ federated.

200. Theo Ryffel, Andrew Trask, Morten Dahl, Bobby Wagner, Jason Mancuso, Daniel Rueckert, and Jonathan PasseratPalmbach. A generic framework for privacy preserving deep learning. arxiv, Nov. 2018. https://arxiv.org/pdf/ 1811.04017.pdf.

201. Qiang Yang, Yang Liu, Tianjian Chen, and Yongxin Tong. Federated machine learning: Concept and applications. ACM Trans. Intell. Syst. Technol., 10(2):12:1-12:19, Feb. 2019.

202. Luca Melis, Congzheng Song, Emiliano De Cristofaro, and Vitaly Shmatikov. Exploiting unintended feature leakage in collaborative learning. In Proc. of 40th IEEE Symp. on Security 8 Privacy, S\& P 2019, pages 691-706, 2019.

203. Matt Fredrikson, Somesh Jha, and Thomas Ristenpart. Model inversion attacks that exploit confidence information and basic countermeasures. In Proc. of the 22Nd ACM SIGSAC Conf. on Comp. and Comm. Security, CCS '15, pages 1322-1333, Oct. 2015.

204. Briland Hitaj, Giuseppe Ateniese, and Fernando Pérez-Cruz. Deep models under the GAN: information leakage from collaborative deep learning. In ACM Conf. on Comp. and Comm. Security, pages 603-618, Oct. 2017.

205. Eugene Bagdasaryan, Andreas Veit, Yiqing Hua, Deborah Estrin, and Vitaly Shmatikov. How to backdoor federated learning. CoRR, abs/1807.00459, 2018.

206. Theodora S. Brisimi, Ruidi Chen, Theofanie Mela, Alex Olshevsky, Ioannis Ch. Paschalidis, and Wei Shi. Federated learning of predictive models from federated electronic health records. I. J. Medical Informatics, 112:59-67, Nov. 2018.

207. Li Huang and Dianbo Liu. Patient clustering improves efficiency of federated machine learning to predict mortality and hospital stay time using distributed electronic medical records. CoRR, abs/1903.09296, Apr. 2019. http://arxiv.org/ abs/1903.09296

208. NVIDIA Developer. Nvidia and kings college london debut first privacy-preserving federated learning system for medical imaging, 2020. https://news.developer.nvidia.com/first-privacy-preserving-federated-learning-system/, last accessed on Jan 28, 2020.

209. Praneeth Vepakomma, Otkrist Gupta, Tristan Swedish, and Ramesh Raskar. Split learning for health: Distributed deep learning without sharing raw patient data. CoRR, Dec. 2018. https://arxiv.org/pdf/1812.00564.pdf.

210. Praneeth Vepakomma, Tristan Swedish, Ramesh Raskar, Otkrist Gupta, and Abhimanyu Dubey. No peek: A survey of private distributed deep learning. CoRR, abs/1812.03288, Dec. 2018. https://arxiv.org/pdf/1812.03288.pdf 
211. Praneeth Vepakomma, Otkrist Gupta, Abhimanyu Dubey, and Ramesh Raskar. Reducing leakage in distributed deep learning for sensitive health data. In The Intl. Conf. on Learning Representations (ICLR) 2019 AI for social Good Workshop, pages 1-6, 2019.

212. Otkrist Gupta and Ramesh Raskar. Distributed learning of deep neural network over multiple agents. Journal of Network and Comp. App., 116:1-8, 2018.

213. Maarten G. Poirot, Praneeth Vepakomma, Ken Chang, Jayashree Kalpathy-Cramer, Rajiv Gupta, and Ramesh Raskar. Split learning for collaborative deep learning in healthcare, 2019.

214. Sharif Abuadbba, Kyuyeon Kim, Minki Kim, Chandra Thapa, Seyit A. Camtepe, Yansong Gao, Hyoungshick Kim, and Surya Nepal. Can we use split learning on $1 \mathrm{~d}$ cnn models for privacy preserving training? In Proc. ACM ASIACCS 2020, pages 1-13, 2020. https://arxiv.org/pdf/2003.12365.pdf.

215. Yansong Gao, Minki Kim, Sharif Abuadbba, Yeonjae Kim, Chandra Thapa, Kyuyeon Kim, Seyit A. Camtepe, Hyoungshick Kim, and Surya Nepal. End-to-end evaluation of federated learning and split learning for internet of things. arxiv, 2020. https://arxiv.org/pdf/2003.13376.pdf.

216. Reza Shokri and Vitaly Shmatikov. Privacy-preserving deep learning. In Proc. of the 22nd ACM SIGSAC Conf. on Comp. and Commm. Security, Denver, CO, USA, October 12-16, 2015, pages 1310-1321, 2015.

217. Priya Goyal, Piotr Dollár, Ross B. Girshick, Pieter Noordhuis, Lukasz Wesolowski, Aapo Kyrola, Andrew Tulloch, Yangqing Jia, and Kaiming He. Accurate, large minibatch SGD: training imagenet in 1 hour. CoRR, abs/1706.02677, Apr. 2017. http://arxiv.org/abs/1706.02677

218. Jianmin Chen, Rajat Monga, Samy Bengio, and Rafal Jozefowicz. Revisiting distributed synchronous sgd. In International Conference on Learning Representations Workshop Track, pages 1-10, 2016. https://arxiv.org/abs/1604.00981.

219. 2014. https://ec.europa.eu/programmes/horizon2020/en/what-horizon-2020, last accessed on Jul $01,2019$.

220. My Health My data team, 2019. http://www.myhealthmydata.eu/, last accessed on Jul 01, 2019.

221. MHMD. Shaping our future. Newsletter 01, Jul. 2018. www.myhealthmydata.eu/wp-content/uploads/2017/10/ MHMD_newsletter_01_DEF_WEB_pag_doppie_110718.pdf, last accessed on Jul 1, 2019.

222. MHMD. Initial list of main requirements. Deliverable 1.1, Oct. 2017. http://www.myhealthmydata.eu/wp-content/ themes/Parallax-One/deliverables/D1.1_Initial-List-of-Main-Requirements.pdf.

223. P. S. Nordholt, N. Volgushev, P. Fauzi, C. Orlandi, P. Scholl, M. Simkin, M. Veeningen, N. Bouman, and Berry Schoenmakers. D1.1 state of the art analysis of mpc techniques and frameworks. H2020-LEIT-ICT 731583 SODA, pages 1-78, Sept. 2017.

224. Paul Koster, Peter Norholt, Tore Frederiksen, and Jonas Lindstrom. D4.2 proof-of-concept for results of tasks 1.2 and 2.2: Implementations of key mpc technologies. H2020-LEIT-ICT 731583 SODA, pages 1-23, Dec. 2018.

225. Mark Abspoel, Sakina Asadova, Frank Blom, Niek J. Bouman, Tore Kasper Frederiksen, Paul Koster, Berry Schoenmakers, Meilof Veeningen, Nikolaj Volgushev, and Niels de Vreede. D2.2 application-oriented mpc protocols. H2020LEIT-ICT 731583 SODA, pages 1-87, Sep. 2018.

226. Sakina Asadova, Niek J. Bouman, Satrajit Ghosh, Paul Koster, Peter Sebastian Nordholt, Claudio Orlandi, Berry Schoenmakers, Peter Scholl, Meilof Veeningen, and Tore Kasper Frederiksen. D1.2 general mpc framework development of novel general-purpose mpc protocols. H2020-LEIT-ICT 731583 SODA, pages 1-82, Oct. 2018.

227. Prastudy Fauzi, Claudio Orlandi, Peter Scholl, Kimberley Thissen, Berry Schoenmakers, Peter van Liesdonk, Meilof Veeningen, and Paul Koster. D3.4 differential privacy and leakage control. H2020-LEIT-ICT 731583 SODA, pages 1-57, Jun. 2019.

228. Mpyc: Secure multiparty computation in python, 2018. https://github.com/lschoe/mpyc, last accessed on Jul 02, 2019.

229. FRESCO, 2019. https://fresco.readthedocs.io/en/latest/index.html last accessed on May 6, 2019.

230. M. Keller, E. Orsini, and P. Scholl. Mascot: Faster malicious arithmetic secure computation with oblivious transfer. In ACM Conf. on Comp. and Comm. Security, pages 830-842, May. 2016.

231. Konfido: Secure and trusted paradigm for interoperable ehealth, 2016. https://konfido-project.eu/ last accessed on Jul 02, 2019.

232. Luigi Coppolino, Salvatore DAntonio, Luigi Romano, and Mariacarla Staffa. Konfido project: A secure infrastructure increasing interoperability on a systemic level among ehealth services across europe. In 2017 IEEE Int. Conf. on Internet of Things (iThings) and IEEE Green Computing and Commun. (GreenCom) and IEEE Cyber, Physical and Social Computing (CPSCom) and IEEE Smart Data (SmartData), pages 342-347, June 2017.

233. A. Theodouli, S. Arakliotis, K. Moschou, K. Votis, and D. Tzovaras. On the design of a blockchain-based system to facilitate healthcare data sharing. In 2018 17th IEEE Int. Conf. On Trust, Security And Privacy In Computing And Communications/ 12th IEEE Int. Conf. On Big Data Science And Engineering (TrustCom/BigDataSE), pages 1374-1379, Aug. 2018.

234. Carlos Ribeiro, Herbert Leitold, Simon Esposito, and David Mitzam. Stork: A real, heterogeneous, large-scale eid management system. Intl. Jour. of Inf. Security, 17, Jul. 2017.

235. Shawn N Murphy, Griffin Weber, Michael Mendis, Vivian Gainer, Henry Chueh, Susanne Churchill, and Isaac Kohane. Serving the enterprise and beyond with informatics for integrating biology and the bedside (i2b2). Journal of the American Med. Info. Assoc.: JAMIA, 17:124-130, Mar. 2010.

236. G. M. Weber, S. N. Murphy, A. J. McMurry, D. MacFadden, D. J. Nigrin, S. Churchill, and I. S. Kohane. The shared health research information network (shrine): A prototype federated query tool for clinical data repositories. Journal of the American Med. Info. Assoc.: JAMIA, 16(05):624-630, 2009. 
237. Eizen Kimura, Koki Hamada, Ryo Kikuchi, Koji Chida, Kazuya Okamoto, Shirou Manabe, Tomohiro Kuroda, Yasushi Matsumura, Toshihiro Takeda, and Naoki Mihara. Evaluation of secure computation in a distributed healthcare setting. 2016 European Fed. for Med. Informatics (EFMI) and IOS Press, pages 152-156, 2016.

238. Chandra Thapa, M.A.P. Chamikara, and Seyit Camtepe. Splitfed: When federated learning meets split learning. arxiv, 2020. https://arxiv.org/pdf/2004.12088.pdf.

239. Milad Nasr, Reza Shokri, and Amir Houmansadr. Comprehensive privacy analysis of deep learning: Passive and active white-box inference attacks against centralized and federated learning. In 2019 IEEE Symp. on Security and Privacy (SP), pages 739-753, May 2019.

240. Olivia Choudhury, Aris Gkoulalas-Divanis, Theodoros Salonidis, Issa Sylla, Yoonyoung Park, Grace Hsu, and Amar Das. Differential privacy-enabled federated learning for sensitive health data. CoRR, abs/1910.02578, 2019.

241. Robin C. Geyer, Tassilo Klein, and Moin Nabi. Differentially private federated learning: A client level perspective. CoRR, abs/1712.07557, 2017.

242. Virginia Smith, Chao-Kai Chiang, Maziar Sanjabi, and Ameet Talwalkar. Federated multi-task learning. In Proc. of the 31st Int. Conf. on Neural Info. Processing Systems, NIPS'17, pages 4424-4434, 2017.

243. Jamie Hayes and Olga Ohrimenko. Contamination attacks and mitigation in multi-party machine learning. In Proc. of the 32Nd Int. Conf. on Neural Info. Processing Systems, NIPS'18, pages 6604-6616, 2018. 